Review

\title{
Brominated Compounds from Marine Sponges of the Genus Aplysina and a Compilation of Their ${ }^{13}$ C NMR Spectral Data
}

Narlize Silva Lira ${ }^{1}$, Ricardo Carneiro Montes ${ }^{1}$, Josean Fechine Tavares ${ }^{1}$,

Marcelo Sobral da Silva ${ }^{1}$, Emidio V. L. da Cunha ${ }^{2}$, Petronio Filgueiras de Athayde-Filho ${ }^{1}$,

Luis Cezar Rodrigues ${ }^{1}$, Celidarque da Silva Dias ${ }^{1, *}$ and Jose Maria Barbosa-Filho ${ }^{1, *}$

1 Laboratory of Pharmaceutical Technology, Federal University of Paraiba, Joao Pessoa 58051-900, PB, Brazil; E-Mails: narlizelira@yahoo.com.br (N.S.L.); ricsony_79@yahoo.com.br (R.C.M.); josean@1tf.ufpb.br (J.F.T.); marcelosobral@1tf.ufpb.br (M.S.d.S.); athayde-filho@quimica.ufpb.br (P.F.d.A.-F.); lcezar@ltf.ufpb.br (L.C.R.)

2 Department of Pharmacy, State University of Paraiba, Campina Grande 58100-000, PB, Brazil; E-Mail: emidio@1tf.ufpb.br

* Authors to whom correspondence should be addressed;

E-Mails: celidarquedias@ltf.ufpb.br(C.d.S.D.); jbarbosa@ltf.ufpb.br (J.M.B.-F);

Tel./Fax: +55-83-32167364 (J.M.B.-F).

Received: 18 August 2011; in revised form: 24 October 2011 / Accepted: 31 October 2011 /

Published: 10 November 2011

Abstract: Aplysina is the best representative genus of the family Aplysinidae. Halogenated substances are its main class of metabolites. These substances contribute greatly to the chemotaxonomy and characterization of the sponges belonging to this genus. Due to their pharmacological activities, these alkaloids are of special interest. The chemistry of halogenated substances and of the alkaloids has long been extensively studied in terrestrial organisms, while the number of marine organisms studied has just started to increase in the last decades. This review describes 101 halogenated substances from 14 species of Aplysina from different parts of the world. These substances can be divided into the following classes: bromotyramines (A), cavernicolins (B), hydroverongiaquinols (C), bromotyrosineketals (D), bromotyrosine lactone derivatives (E), oxazolidones (F), spiroisoxazolines (G), verongiabenzenoids (H), verongiaquinols (I), and dibromocyclohexadienes (J). A compilation of their ${ }^{13} \mathrm{C} N M R$ data is also part of the review. For this purpose 138 references were consulted.

Keywords: Aplysina; marine sponges; halogenated substances; ${ }^{13} \mathrm{C}$ NMR; review 


\section{Introduction}

Marine sponges have been known and used by mankind since antiquity. They were included in the first classification of living organisms, written in $350 \mathrm{BC}$ by Aristotle in Greece. At first thought to be plants, their animal nature was only recognized by the end of the XVIII century. However, great naturalists of the time such as Lamarck, Linnaeus and Cuvier classified them as Zoophytes. The elevation of the Porifera to the level of phylum was suggested by Huxley in 1875 and by Sollas in 1884 , and was only accepted at the beginning of the XX century [1].

Sponges belong to the phylum Porifera and are the most primitive of multicelled animals, having existed for roughly $700-800$ million years. They have a very simple physiology of construction. They are aquatic organisms growing mostly in temperate salt waters but may also be found in fresh water. When reaching adult form, they are found in solid substrates in places that allow adequate conditions for their growth. Some, when in their primary states, may be mobile [2-4]. They are easily found in all marine environments, from the intertidal zones to the ocean depths of $8500 \mathrm{~m}$ in tropical and polar seas. Despite their wide distribution in terms of different oceans and depths, the rocky non-polluted coastline areas show greater populations of sponges which are also known for being rich in secondary metabolites [5-9].

The sponges are filtering animals, which utilize flagellate cells called coenocytes for promoting the circulation of the water through a system of canals existing in this phylum only called aquifer system, around which their body is built. This water flow brings organic particles and microorganisms which are filtered and eaten [10]. Of all the known sponges, only 1\% grow in fresh water [11].

There are basically three classes of sponges, Calcarea (5 orders and 24 families), Desmospongiae (15 orders and 92 families) and Hexactinellida (6 orders and 20 families). So far, about 15,000 species of sponges have been described, their diversity however is believed to be much bigger than this $[4,12]$. Being sessile simple organisms, they evolved chemical defense mechanisms to protect themselves against predators and competitors, as well as against infectious microorganisms. Studies show that secondary metabolites in sponges carry out a crucial role in their survival in the marine ecosystem $[13,14]$.

Because of their potential for the production of new substances of pharmacological interest, sponges have been one of the most chemically studied organisms. In the past 20 years, hundreds of substances have been isolated from them and many of those substances have already been identified, and present interesting biological and pharmacological (such as antibacterial, anticoagulant, antifungal, antimalarial, antituberculosis, antiviral, immunosuppressive and neuro-suppressor) activities [15-23]. The main reported activities for the Aplysina genus are antibacterial, antiyeast, antifungal, antiviral, cytotoxic and hyperglycemic activities, which can be seen in Table 1. 
Table 1. Bioactivities of marine sponges of the Aplysina genus.

\begin{tabular}{|c|c|c|c|c|}
\hline Activity/Species Name & Type of Extract & Bioassays Models, Organism, Dose or Route of Administration & Result & Ref. \\
\hline \multicolumn{5}{|l|}{ Antibacterial activity } \\
\hline Aplysina archeri & MeOH Ext. & Agar plate-Bacillus subtilis; Escherichia coli-1.0 mg/Disc & Active & [24] \\
\hline \multirow[t]{3}{*}{ Aplysina fistularis } & MeOH Ext. & Agar plate-Bacillus subtilis; Escherichia coli & Active & [25] \\
\hline & MeOH-Toluene & Agar plate-Bacillus subtilis; Escherichia coli & Active & [26] \\
\hline & Chromatographic Fraction & $\begin{array}{l}\text { Agar plate-Staphylococcus aureus; Sarcinalutea; Klebsiella } \\
\text { pneumonia; Proteus vulgaris; Bacteroides fragilis; Clostridium } \\
\text { perfringens; Mycobacterium aviun }\end{array}$ & Active & [26] \\
\hline \multirow[t]{2}{*}{ Aplysina lacunosa } & MeOH Ext. & Agar plate-Bacillus subtilis; Escherichia coli & Active & [25] \\
\hline & MeOH Ext. & Agar plate-Bacillus subtilis; Escherichia coli-1.0 mg/Disc & Active & [24] \\
\hline Aplysina laevis & Acetone Ext. & Agar plate-Bacillus subtilis; Escherichia coli & Active & [27] \\
\hline \multirow[t]{7}{*}{ Aplysina mollis } & Ether Ext. & Agar plate-Staphylococcus aureus- $0.2 \mu \mathrm{L} / \mathrm{Disc}$ & Active & [28] \\
\hline & Ether Ext. & Agar plate-Escherichia coli; Pseudomonas aeruginosa- $0.2 \mu \mathrm{L} /$ Disc & Inactive & [28] \\
\hline & $\mathrm{CHCl}_{3}$ Ext. & Agar plate-Staphylococcus aureus- $0.2 \mu \mathrm{L} / \mathrm{Disc}$ & Active & [28] \\
\hline & $\mathrm{CHCl}_{3}$ Ext. & Agar plate-Escherichia coli; Pseudomonas aeruginosa- $0.2 \mu \mathrm{L} /$ Disc & Inactive & [28] \\
\hline & Acetone Ext. & Agar plate-Staphylococcus aureus- $0.2 \mu \mathrm{L} / \mathrm{Disc}$ & Inactive & [28] \\
\hline & ETOH (95\%) Ext. & $\begin{array}{l}\text { Agar plate-Staphylococcus aureus; Escherichia coli; Pseudomonas } \\
\text { aeruginosa-0.2 } \mu \mathrm{L} / \mathrm{Disc}\end{array}$ & Inactive & [28] \\
\hline & Benzene Ext. & $\begin{array}{l}\text { Agar plate-Staphylococcus aureus; Escherichia coli; Pseudomonas } \\
\text { aeruginosa- } 0.2 \mu \mathrm{L} / \mathrm{Disc}\end{array}$ & Inactive & [28] \\
\hline \multirow[t]{3}{*}{ Aplysina species } & Ether Ext. & $\begin{array}{l}\text { Agar plate-Staphylococcus aureus; Escherichia coli; Pseudomonas } \\
\text { aeruginosa- } 0.2 \mu \mathrm{L} / \mathrm{Disc}\end{array}$ & Inactive & [28] \\
\hline & Acetone Ext. & $\begin{array}{l}\text { Agar plate-Staphylococcus aureus; Escherichia coli; Pseudomonas } \\
\text { aeruginosa- } 0.2 \mu \mathrm{L} / \mathrm{Disc}\end{array}$ & Inactive & [28] \\
\hline & ETOH (95\%) Ext. & $\begin{array}{l}\text { Agar plate-Staphylococcus aureus; Escherichia coli; Pseudomonas } \\
\text { aeruginosa- } 0.2 \mu \mathrm{L} / \mathrm{Disc}\end{array}$ & Inactive & [28] \\
\hline
\end{tabular}


Table 1. Cont.

\begin{tabular}{|c|c|c|c|c|}
\hline $\begin{array}{l}\text { Antibacterial activity } \\
\text { Aplysina species }\end{array}$ & $\mathrm{CHCl}_{3}$ Ext. & $\begin{array}{l}\text { Agar plate-Staphylococcus aureus; Escherichia coli; Pseudomonas } \\
\text { aeruginosa- } 0.2 \mu \mathrm{L} / \text { Disc } \\
\text { Agar plate-Staphylococcus aureus; Escherichia coli; Pseudomonas } \\
\text { aeruginosa- } 0.2 \mu \mathrm{L} / \text { Disc }\end{array}$ & Inactive & [28] \\
\hline $\begin{array}{l}\text { Antiyeast activity } \\
\text { Aplysina archeri } \\
\text { Aplysina lacunosa }\end{array}$ & $\begin{array}{l}\text { MeOH Ext. } \\
\text { MeOH Ext. }\end{array}$ & $\begin{array}{l}\text { Agar plate-Saccharomyces cerevisiae- } 1.0 \mathrm{mg} / \mathrm{Disc} \\
\text { Agar plate-Saccharomyces cerevisiae-1.0 mg/Disc }\end{array}$ & $\begin{array}{l}\text { Inactive } \\
\text { Weak } \\
\text { Activity }\end{array}$ & $\begin{array}{l}{[24]} \\
{[24]}\end{array}$ \\
\hline $\begin{array}{l}\text { Antifungal activity } \\
\text { Aplysina archeri } \\
\text { Aplysina lacunosa }\end{array}$ & $\begin{array}{l}\text { MeOH Ext. } \\
\text { MeOH Ext. }\end{array}$ & $\begin{array}{l}\text { Agar plate-Penicillium atrovenetum-1.0 mg/Disc } \\
\text { Agar plate-Penicillium atrovenetum-1.0 mg/Disc }\end{array}$ & $\begin{array}{l}\text { Inactive } \\
\text { Inactive }\end{array}$ & $\begin{array}{l}{[24]} \\
{[24]}\end{array}$ \\
\hline $\begin{array}{l}\text { Antiviral activity } \\
\text { Aplysina archeri }\end{array}$ & MeOH-Toluene & Cell culture-Virus-Feline Leukemia & Active & [29] \\
\hline $\begin{array}{l}\text { Cytotoxic activity } \\
\text { Aplysina cauliformis } \\
\text { Aplysina fistularis }\end{array}$ & $\begin{array}{l}\mathrm{CHCl}_{3}-\mathrm{MeOH} \text { Ext. (1:1) } \\
\text { MeOH Ext. } \\
\text { MeOH-Toluene } \\
\text { Chromatographic Fraction } \\
\text { Isopropanol Ext. } \\
\text { Isopropanol Ext. } \\
\text { Isopropanol Ext. } \\
\text { MeOH Ext. }\end{array}$ & $\begin{array}{l}\text { Cell culture-Cells-Cho K-1 } \\
\text { Cell culture-Leuk L-1210-ED } 5050 \mathrm{mcg} / \mathrm{mL} \\
\text { Cell culture-CA-9KB } \\
\text { Cell culture-Leuk } L-1210-\mathrm{IC}_{50} 0.14 \mathrm{mcg} / \mathrm{mL} \\
\text { Cell culture-CA-9KB }<\mathrm{ED}_{50} 20 \mathrm{mcg} / \mathrm{mL} \\
\text { Cell culture-Leuk } L-1210<\mathrm{ED}_{50} 20 \mathrm{mcg} / \mathrm{mL} \\
\text { Cell culture-Leuk } P-388<\mathrm{ED}_{50} 20 \mathrm{mcg} / \mathrm{mL} \\
\text { Cell culture-Leuk } L-1210-\mathrm{ED}_{50} 8.2 \mathrm{mcg} / \mathrm{mL}\end{array}$ & $\begin{array}{l}\text { Active } \\
\text { Active } \\
\text { Active } \\
\text { Active } \\
\text { Active } \\
\text { Active } \\
\text { Active } \\
\text { Inactive }\end{array}$ & $\begin{array}{l}{[30]} \\
{[25]} \\
{[26]} \\
{[26]} \\
{[31]} \\
{[31]} \\
{[31]} \\
{[25]}\end{array}$ \\
\hline $\begin{array}{l}\text { Hyperglycemic activity } \\
\text { Aplysina mollis }\end{array}$ & ETOH $(95 \%)$ Ext. & Mouse-Intragastric-Dose $200 \mathrm{mg} / \mathrm{kg}$ & Active & [32] \\
\hline
\end{tabular}


The pioneer investigative work in the field of sponge chemistry published by Bergmann and Feeney in the beginning of the 1950s led to the discovery of Cryptotethya crypta bioactive nucleosides spongothymidine and spongouridine [21]. These nucleosides were the basis for the synthesis of Ara-C, the first marine derivative anticancer agent, and antiviral drug Ara-A [22]. Today, Ara-C is used in the routine treatment of patients suffering from leukemia and lymphomas. One of its derivatives was also approved for use in patients with cancer of the pancreas, lungs and breast [23].

\section{The Genus Aplysina}

The genus Aplysina, formerly known as Verongia and reclassified to Aplysina, is one of the richest in terms of secondary metabolites, described in 14 species of the family Aplysinidae, there are 2 species from the Mediterranean Sea, 8 from the Caribbean, 3 from the Pacific Coast of Mexico and 15 in the Brazilian coast. Of the above species, 8 have only been recently identified. From the Mediterranean Sea, the two described species of the genus Aplysina are: A. aerophoba (Schmidt, 1862) and $A$. cavernicola (Vacelet, 1959). From the Caribbean, among others we find A. fistularis insularis, A. fistularis form fulva, A. archeri, A. cauliformis and A. Lacunosa [33].

Like other genera of the order Verongida, Aplysina stands out for its unique biochemical characteristics. They show low terpene content, and possess a moderately high percentage of sterols, mostly within the aplystan skeleton. They also produce a significant series of brominated derivatives of tyrosine metabolites considered peculiar to species of this order. The sponges of this order are also known for their high phenotypic variability [34].

Marine organisms produce a cocktail of halogenated metabolites with potential commercial value. The structures found in these compounds go from linear chain acyclic, to complex polycyclic molecules [35,36]. The research of halogenated metabolites has been more focused on marine algae than on sea sponges [37-41]. Though many compounds have been discovered recently, many sponges species are poorly screened and the need for new drugs keeps this field open.

In a previous paper our research group evaluated crude algae, sponge extracts and chemically determined molecules from Northeastern Brazil [42-48] with database survey [49-62].

In this paper we review halogenated substances from the genus Aplysina. A compilation of the

${ }^{13} \mathrm{C}$ NMR spectral data of the selected natural products is also provided. This type of genus and species investigation is helpful in the identification and capture of halogenated substances from the genus.

\section{Methodology}

An extensive bibliographic review was carried out to identify studies of halogenated substances isolated from the genus Aplysina. The present review covers the period of 1967 thru 2010. The search was performed using the following databases: NAPRALERT (Natural Products Alert at the University of Illinois, Chicago), Chemical Abstracts, and the Brazilian online scientific literature search system called "Periodical CAPES" (Coordination for the Improvement of Graduate Level Personnel).

Tables 2 and 3 respectively show the halogenated substance distribution in the genus Aplysina, and the basic skeletons of those substances. Table 4 shows the different substituents for the diverse classes of halogenated substances. Table 5 describes the position of the substituents for the 101 substances isolated from each species. Finally Tables 6-14 show a compilation of ${ }^{13} \mathrm{C}$ NMR data of the substances. 
Table 2. Distribution of the halogenated substances in the genus Aplysina.

\begin{tabular}{|c|c|c|c|c|}
\hline Species & Halogenated Substances & Substance Code & Nucleus & Ref. \\
\hline \multirow{13}{*}{$\begin{array}{c}\text { A. aerophoba } \\
\text { (Schmidt, 1862) }\end{array}$} & Aeroplysinine 2 & 25 & $\mathrm{E}_{1}$ & [63] \\
\hline & Aplysinadiene & 26 & $\mathrm{E}_{1}$ & [64] \\
\hline & $\begin{array}{l}\left(7 S^{*}, 11 R^{*}\right)-5-[3,5 \text {-Dibromo-4-[(2-oxo-5-oxazolidinyl)]methoxyphenyl]-2- } \\
\text { oxazolidinone }\end{array}$ & 29 & $\mathrm{~F}_{1}$ & {$[65]$} \\
\hline & $\begin{array}{l}(R, R)-5[3,5 \text {-Dibromo-4-[(2-oxo-5-oxazolidinyl })] \text { methoxyphenyl]-2- } \\
\text { oxazolidone }\end{array}$ & 31 & $\mathrm{~F}_{1}$ & {$[64]$} \\
\hline & Aerothionin & 41 & $\mathrm{G}_{1}$ & [34] \\
\hline & Homoaerothionin & 47 & $\mathrm{G}_{2}$ & [66] \\
\hline & Isofistularin-3 & 48 & $\mathrm{G}_{2}$ & [67] \\
\hline & Aerophobin-1 & 76 & $\mathrm{G}_{7}$ & [68] \\
\hline & 2-(3,5-Dibromo-2-hydroxy-4-methoxyphenyl) acetamide & 84 & $\mathrm{H}$ & [69] \\
\hline & 3,5-Dibromo-4-methoxyphenol & 85 & $\mathrm{H}$ & [64] \\
\hline & Methyl 2-(3,5-dibromo-2-hydroxy-4-methoxyphenyl) acetate & 86 & $\mathrm{H}$ & [69] \\
\hline & $\begin{array}{l}\text { Dibromoverongiaquinol or dienone or } \\
\text { 3-5-dibromo-1-hydroxy-4-oxocyclohexa-2-5-diene-1-acetamide }\end{array}$ & 94 & $\mathrm{I}_{1}$ & {$[64]$} \\
\hline & Aeroplysinin 1 & 100 & $\mathrm{~J}$ & {$[66,70]$} \\
\hline \multirow{7}{*}{$\begin{array}{c}\text { A. archeri } \\
\text { (Higgin, 1875) }\end{array}$} & $\begin{array}{l}\left(7 S^{*}, 11 R^{*}\right)-5-[3,5-D i b r o m o-4-[(2-o x o-5-o x a z o l i d i n y l)] \text { methoxyphenyl]-2- } \\
\text { oxazolidinone }\end{array}$ & 29 & $\mathrm{~F}_{1}$ & [71] \\
\hline & 11,19-Dideoxyfistularin 3 & 33 & $\mathrm{G}_{1}$ & [72] \\
\hline & Archerine & 43 & $\mathrm{G}_{1}$ & [73] \\
\hline & Fistularin-3 & 46 & $\mathrm{G}_{2}$ & [29] \\
\hline & 11-Ketofistularin 3 & 51 & $\mathrm{G}_{2}$ & [29] \\
\hline & $\begin{array}{l}\text { Aplysina compound } 1 \text { or 1-Oxa-2-azaspiro[4,5]deca-2,6-diene-3-carboxamide, } \\
N, N^{\prime}-(2 \text {-oxo-1,4-butanediyl)bis[7,9-dibromo-10-hydroxy-8-oxo, }[5 R- \\
\left.\left[5 \alpha\left(5^{\prime} R^{*}, 9^{\prime} R^{*}, 10^{\prime} S^{*}\right), 9 \alpha, 10 \beta\right]\right]-(9 \mathrm{CI})\end{array}$ & 57 & $\mathrm{G}_{4}$ & {$[74]$} \\
\hline & Aeroplysinin 1 & 100 & $\mathrm{~J}$ & [74] \\
\hline
\end{tabular}


Table 2. Cont.

\begin{tabular}{|c|c|c|c|c|}
\hline \multirow{2}{*}{$\begin{array}{c}\text { A. archeri } \\
\text { (Higgin, 1875) }\end{array}$} & $(+)$ Aeroplysinin 1 & 100 & $\mathrm{~J}$ & [71] \\
\hline & (-) Aeroplysinin 1 & 100 & $\mathrm{~J}$ & [71] \\
\hline \multirow{8}{*}{$\begin{array}{l}\text { A. caissara } \\
\text { (Pinheiro \& } \\
\text { Hajdu, 2001) }\end{array}$} & 2-(3,5-Dibromo-4,4-dimethoxy-1-hydroxy-2,5-cyclohexadien-1-yl) acetamide & 21 & $\mathrm{D}_{1}$ & [75] \\
\hline & Caissarine C & 42 & $\mathrm{G}_{1}$ & [76] \\
\hline & Caissarine B & 53 & $\mathrm{G}_{2}$ & [75] \\
\hline & Agelocaissarine A1 & 58 & $\mathrm{G}_{4}$ & [76] \\
\hline & Agelocaissarine A2 & 59 & $\mathrm{G}_{4}$ & [76] \\
\hline & Agelocaissarine B1 & 60 & $\mathrm{G}_{4}$ & [76] \\
\hline & Agelocaissarine B2 & 61 & $\mathrm{G}_{4}$ & [76] \\
\hline & Caissarine A & 79 & $\mathrm{G}_{8}$ & [75] \\
\hline \multirow{10}{*}{$\begin{array}{l}\text { A. cauliformis } \\
\text { (Carter, 1882) }\end{array}$} & 2-(3,5-Dibromo-1-hydroxy-4,4-dimethoxycyclohexa-2,5-dienyl)acetamide & 21 & $\mathrm{D}_{1}$ & [71] \\
\hline & $\begin{array}{l}\left.\left(7 S^{*}, 11 R^{*}\right)-5-[3,5 \text {-Dibromo-4-[(2-oxo-5-oxazolidinyl })\right] \text { methoxyphenyl]-2- } \\
\text { oxazolidinone }\end{array}$ & 29 & $\mathrm{~F}_{1}$ & [71] \\
\hline & 11-Oxoaerothionin & 52 & $\mathrm{G}_{2}$ & [77] \\
\hline & $\begin{array}{l}\text { Aplysinametabolite or Methyl 4-((5S,10R)-7,9-dibromo-10-hydroxy-8- } \\
\text { methoxy-1-oxa-2-azaspiro-[4.5]deca-2,6,8-trienecarboxamido)-2- } \\
\text { oxobutylcarbamate }\end{array}$ & 64 & $\mathrm{G}_{7}$ & [78] \\
\hline & $\begin{array}{l}\text { Methyl 4-((5S,10R)-7,9-dibromo-10-hydroxy-8-methoxy-1-oxa-2-azaspiro- } \\
\text { [4.5]deca-2,6,8-trienecarboxamido)-3-oxobutylcarbamate or Aplysina } \\
\text { compound } 13\end{array}$ & 65 & $\mathrm{G}_{7}$ & [78] \\
\hline & $\begin{array}{l}\text { Methyl-4-((5S,10R)-7,9-dibromo-10-hydroxy-8-methoxy-1-oxa-2- } \\
\text { azaspiro[4.5]deca-2,6,8-trienecarboxamido)butylcarbamate or Aplysina } \\
\text { metabolite } 14\end{array}$ & 66 & $\mathrm{G}_{7}$ & [78] \\
\hline & Aplysinamisine-1 & 67 & $\mathrm{G}_{7}$ & [30] \\
\hline & Aplysinamisine-2 & 68 & $\mathrm{G}_{7}$ & [30] \\
\hline & Aplysinamisine-3 & 69 & $\mathrm{G}_{7}$ & [30] \\
\hline & Aeroplysinin 1 & 100 & $\mathrm{~J}$ & [78] \\
\hline
\end{tabular}


Table 2. Cont

\begin{tabular}{|c|c|c|c|c|}
\hline \multirow{24}{*}{$\begin{array}{l}\text { A. cavernicola } \\
\text { (Vacelet, 1959) }\end{array}$} & Cavernicolin-1 & 5 & $\mathrm{~B}_{1}$ & {$[79,80]$} \\
\hline & 5-Bromo-7 $\alpha$-chlorocavernicolin & 7 & $\mathrm{~B}_{2}$ & {$[81]$} \\
\hline & 5-Bromo-7 $\beta$-chlorocavernicolin & 8 & $\mathrm{~B}_{2}$ & [81] \\
\hline & 7 $\beta$-Bromo-5-chlorocavernicolin & 9 & $\mathrm{~B}_{2}$ & [81] \\
\hline & 7 $\alpha$-Bromo-5-chlorocavernicolin & 10 & $\mathrm{~B}_{2}$ & [81] \\
\hline & Monobromocavernicolin or 5-Bromocavernicolin & 11 & $\mathrm{~B}_{2}$ & {$[81]$} \\
\hline & 5-Chlorocavernicolin & 12 & $\mathrm{~B}_{2}$ & {$[81,82]$} \\
\hline & 7-Bromocavernicolenone & 13 & $\mathrm{~B}_{3}$ & {$[82]$} \\
\hline & 7-Chlorocavernicolenone & 14 & $\mathrm{~B}_{3}$ & {$[63]$} \\
\hline & 2-(3,5-Dibromo-1-hydroxy-4,4-dimethoxycyclohexa-2,5-dienyl) acetamide & 21 & $\mathrm{D}_{1}$ & {$[63]$} \\
\hline & Aeroplysinine 2 & 25 & $E_{1}$ & [63] \\
\hline & 11,19-Dideoxyfistularin 3 & 33 & $\mathrm{G}_{1}$ & {$[63]$} \\
\hline & 12(R)-Hydroxy-11-oxoaerothionin & 36 & $\mathrm{G}_{1}$ & [63] \\
\hline & Aerothionin & 41 & $\mathrm{G}_{1}$ & {$[63]$} \\
\hline & Oxohomoaerothionin & 44 & $\mathrm{G}_{1}$ & [63] \\
\hline & 11-Deoxyfistularin-3 & 45 & $\mathrm{G}_{2}$ & {$[63]$} \\
\hline & Homoaerothionin & 47 & $\mathrm{G}_{2}$ & {$[63]$} \\
\hline & Isofistularin3 & 48 & $\mathrm{G}_{2}$ & {$[63]$} \\
\hline & 11-Oxoaerothionin & 52 & $\mathrm{G}_{2}$ & [63] \\
\hline & $\begin{array}{l}\text { (+) 3-Bromo-5-chloroverongiaquinol or }(+) \text {-3-Bromo-5-chloro-1-hydroxy-4- } \\
\text { oxo-2,5-cyclohexadiene-1-acetamide }\end{array}$ & 91 & $\mathrm{I}_{1}$ & {$[81]$} \\
\hline & $\begin{array}{l}(+) \text { 3-Bromoverongiaquinol or }(+)-3 \text {-Bromo-1-hydroxy-4-oxo-2,5- } \\
\text { cyclohexadiene-1-acetamide }\end{array}$ & 92 & $\mathrm{I}_{1}$ & {$[81]$} \\
\hline & (DL) 5-Bromoverongiaquinol & 93 & $\mathrm{I}_{1}$ & [81] \\
\hline & Dichloroverongiaquinol & 95 & $\mathrm{I}_{1}$ & {$[80]$} \\
\hline & Aeroplysinin 1 & 100 & $\mathrm{~J}$ & [63] \\
\hline
\end{tabular}


Table 2. Cont

\begin{tabular}{|c|c|c|c|c|}
\hline $\begin{array}{c}\text { A. conulosa } \\
\text { (Pulitzer-Finali, } \\
\text { 1986) }\end{array}$ & Aeroplysinine 2 & 25 & $\mathrm{E}_{1}$ & [83] \\
\hline \multirow{20}{*}{$\begin{array}{l}\text { A. fistularis } \\
\text { (Pallas, 1766) }\end{array}$} & Aplysamine1 & 1 & A & {$[84]$} \\
\hline & Aplysamine2 & 2 & A & [84] \\
\hline & Aplysfistularine & 4 & A & [46] \\
\hline & 5-Amino-2,6-dichloro-4-hydroxycyclohex-2-enone acetic acid lactam & 15 & $\mathrm{~B}_{4}$ & {$[26]$} \\
\hline & $\begin{array}{l}\text { 5-Amino-2-bromo-6-chloro-4-hydroxy-cyclohex-2-enone acetic acid lactam } \\
\text { (5-bromo-7-chlorocavernicolin) }\end{array}$ & 16 & $\mathrm{~B}_{4}$ & {$[26]$} \\
\hline & $\begin{array}{l}\text { 5-Amino-2-6-dibromo-4-hydroxy-cyclohex-2-enone acetic acid lactam or } \\
\text { Cavernicolin }\end{array}$ & 17 & $\mathrm{~B}_{4}$ & {$[26]$} \\
\hline & 4,6-Dibromohomogentisamide & 18 & $\mathrm{C}$ & {$[85]$} \\
\hline & 3,5-Dibromohydroquinone-2-acetamide & 19 & $\mathrm{C}$ & {$[86]$} \\
\hline & 2-(3,5-Dibromo-1-hydroxy-4,4-dimethoxycyclohexa-2,5-dienyl) acetamide & 21 & $\mathrm{D}_{1}$ & [87] \\
\hline & Aeroplysinine 2 & 25 & $\mathrm{E}_{1}$ & {$[88]$} \\
\hline & $\begin{array}{l}\left.\left(7 S^{*}, 11 R^{*}\right)-5-[3,5-\text { Dibromo-4-[(2-oxo-5-oxazolidinyl })\right] \text { methoxyphenyl]-2- } \\
\text { oxazolidinone }\end{array}$ & 29 & $\mathrm{~F}_{1}$ & [71] \\
\hline & Aerothionin & 41 & $\mathrm{G}_{1}$ & {$[88,89]$} \\
\hline & Fistularin-3 & 46 & $\mathrm{G}_{2}$ & {$[88]$} \\
\hline & Homoaerothionin & 47 & $\mathrm{G}_{2}$ & [89] \\
\hline & 11-Oxoaerothionin & 52 & $\mathrm{G}_{2}$ & [88] \\
\hline & Purealidin-L & 78 & $\mathrm{G}_{7}$ & {$[90]$} \\
\hline & 2-(3,5-Dibromo-2-hydroxy-4-methoxyphenyl) acetamide & 84 & $\mathrm{H}$ & {$[26,69]$} \\
\hline & 2,6-Dibromo-1,4-benzoquinone & 87 & $\mathrm{I}_{1}$ & [91] \\
\hline & 2,6-Dichloro-4-hydroxycyclohexa-2-5-dienone-4-acetamide & 89 & $\mathrm{I}_{1}$ & [26] \\
\hline & 2-Bromo-6-chloro-4-hydroxycyclohexa-2,5-dienone-4-acetamide & 90 & $\mathrm{I}_{1}$ & {$[26]$} \\
\hline
\end{tabular}


Table 2. Cont.

\begin{tabular}{|c|c|c|c|c|}
\hline \multirow[t]{2}{*}{$\begin{array}{l}\text { A. fistularis } \\
\text { (Pallas, 1766) }\end{array}$} & $\begin{array}{l}\text { Dibromoverongiaquinol or dienone or 3-5-dibromo-1-hydroxy-4- } \\
\text { oxocyclohexa-2-5-diene-1-acetamide }\end{array}$ & 94 & $\mathrm{I}_{1}$ & {$[61-65,92-96]$} \\
\hline & Aeroplysinin 1 & 100 & $\mathrm{~J}$ & {$[96]$} \\
\hline \multirow{22}{*}{$\begin{array}{l}\text { A. fulva } \\
\text { (Pallas, 1766) }\end{array}$} & Cavernicolin-1 & 5 & $\mathrm{~B}_{1}$ & {$[83,97]$} \\
\hline & Cavernicolin-2 & 6 & $\mathrm{~B}_{1}$ & {$[83,97]$} \\
\hline & 3,5-Dibromohydroquinone-2-acetamide & 19 & $\mathrm{C}$ & [34] \\
\hline & 2'-(3,5-Dibromo-4-hydroxyphenyl) acetamide & 20 & $\mathrm{C}$ & [97] \\
\hline & 2-(3,5-Dibromo-1-hydroxy-4,4-dimethoxycyclohexa-2,5-dienyl) acetamide & 21 & $\mathrm{D}_{1}$ & [97] \\
\hline & Aeroplysinine 2 & 25 & $\mathrm{E}_{1}$ & {$[34]$} \\
\hline & $\begin{array}{l}\left.\left(7 S^{*}, 11 R^{*}\right)-5-[3,5-\text { Dibromo-4-[(2-oxo-5-oxazolidinyl })\right] \text { methoxyphenyl]-2- } \\
\text { oxazolidinone }\end{array}$ & 29 & $\mathrm{~F}_{1}$ & {$[97]$} \\
\hline & 11-Epi-fistularin-3 & 34 & $\mathrm{G}_{1}$ & [98] \\
\hline & 11-Hydroxyfistularin-3 & 35 & $\mathrm{G}_{1}$ & [99] \\
\hline & 12(R)-Hydroxy-11-oxoaerothionine & 36 & $\mathrm{G}_{1}$ & {$[34]$} \\
\hline & 12(S)-Hydroxy-11-oxoaerothionine & 37 & $\mathrm{G}_{1}$ & {$[34]$} \\
\hline & Aerothionin & 41 & $\mathrm{G}_{1}$ & {$[34,83,97]$} \\
\hline & Fistularin-3 & 46 & $\mathrm{G}_{2}$ & {$[31,34,83,97]$} \\
\hline & Homoaerothionin & 47 & $\mathrm{G}_{2}$ & [34] \\
\hline & 11-Hydroxyaerothionin & 50 & $\mathrm{G}_{2}$ & {$[34,97]$} \\
\hline & 11-Oxoaerothionin & 52 & $\mathrm{G}_{2}$ & {$[34,97]$} \\
\hline & Aplysinamisine-1 & 67 & $\mathrm{G}_{7}$ & {$[97]$} \\
\hline & Araplysillin $N^{9}$-sulfamate & 70 & $\mathrm{G}_{7}$ & [99] \\
\hline & Fistularin-1 & 72 & $\mathrm{G}_{7}$ & {$[34]$} \\
\hline & Fistularin-2 & 73 & $\mathrm{G}_{7}$ & [31] \\
\hline & $\begin{array}{l}N \text {-[5S,10R)-7,9-Dibromo-10-hydroxy-8-methoxy-1-oxa-2-azaspiro[4.5]deca- } \\
\text { 2,6,8-triene-3-carboxy]-4-aminobutanoic acid }\end{array}$ & 74 & $\mathrm{G}_{7}$ & [99] \\
\hline & Aerophobin-1 & 76 & $\mathrm{G}_{7}$ & [97] \\
\hline
\end{tabular}


Table 2. Cont

\begin{tabular}{|c|c|c|c|c|}
\hline \multirow{3}{*}{$\begin{array}{c}\text { A. fulva } \\
\text { (Pallas, 1766) }\end{array}$} & Aerophobin-2 & 77 & $\mathrm{G}_{7}$ & [97] \\
\hline & Aeroplysinin 1 & 100 & $\mathrm{~J}$ & {$[34,66]$} \\
\hline & Aplysinafulvin & 101 & $\mathrm{~J}$ & {$[97]$} \\
\hline \multirow{8}{*}{$\begin{array}{l}\text { A. gerardogreeni } \\
\text { (Gomes \& } \\
\text { Bakus, 1992) }\end{array}$} & Aerothionin & 41 & $\mathrm{G}_{1}$ & {$[100]$} \\
\hline & Homoaerothionin & 47 & $\mathrm{G}_{2}$ & [101] \\
\hline & Aplysinone A & 54 & $\mathrm{G}_{3}$ & [101] \\
\hline & Aplysinone D & 55 & $\mathrm{G}_{3}$ & {$[101]$} \\
\hline & Aplysinone B & 56 & $\mathrm{G}_{4}$ & {$[101]$} \\
\hline & Calafianin & 62 & $\mathrm{G}_{5}$ & {$[100]$} \\
\hline & Aplysinone C & 63 & $\mathrm{G}_{6}$ & [101] \\
\hline & 2-(3,5-dibromo-2-hydroxy-4-methoxyphenyl) acetic acid & 83 & $\mathrm{H}$ & {$[100]$} \\
\hline \multirow{13}{*}{$\begin{array}{c}\text { A. insularis } \\
\text { (Duchassaing \& } \\
\text { Michelotti, } \\
\text { 1864) }\end{array}$} & $\begin{array}{l}\text { 5-((2,6-Dibromo-4-(2-oxooxazolidin-5-yl)-phenoxy)-methyl)-5- } \\
\text { methoxyoxazolidin-2-one }\end{array}$ & 32 & $\mathrm{~F}_{3}$ & {$[74]$} \\
\hline & 11,19-Dideoxyfistularin 3 & 33 & $\mathrm{G}_{1}$ & [102] \\
\hline & Aerothionin & 41 & $\mathrm{G}_{1}$ & {$[74,103]$} \\
\hline & Fistularin-3 & 46 & $\mathrm{G}_{2}$ & {$[74,102,103]$} \\
\hline & Homoaerothionin & 47 & $\mathrm{G}_{2}$ & {$[103]$} \\
\hline & 11-Dihydroaerothionin & 49 & $\mathrm{G}_{2}$ & [102] \\
\hline & 11-Oxoaerothionin & 52 & $\mathrm{G}_{2}$ & {$[102,103]$} \\
\hline & Aplysina metabolite 14 & 66 & $\mathrm{G}_{7}$ & {$[74]$} \\
\hline & 14-Oxoaerophobin-2 & 75 & $\mathrm{G}_{7}$ & [102] \\
\hline & Aerophobin-1 & 76 & $\mathrm{G}_{7}$ & {$[102]$} \\
\hline & Aerophobin-2 & 77 & $\mathrm{G}_{7}$ & [102] \\
\hline & $\begin{array}{l}\text { (5S,10R)-Methyl 7,9-dibromo-10-hydroxy-8-methoxy-1-oxa-2- } \\
\text { azaspiro[4.5]deca-2,6,8-triene-3-carboxylate }\end{array}$ & 80 & $\mathrm{G}_{9}$ & {$[102]$} \\
\hline & 2-(3-Dibromo-4-hydroxyphenyl)- $N, N, N$-trimethylethanaminium & 82 & $\mathrm{H}$ & {$[102]$} \\
\hline
\end{tabular}


Table 2. Cont.

\begin{tabular}{|c|c|c|c|c|}
\hline \multirow{7}{*}{$\begin{array}{l}\text { A. lacunose } \\
\text { (Lamarck, 1814) }\end{array}$} & $\begin{array}{l}\left(7 S^{*}, 11 R^{*}\right)-5-[3,5-D i b r o m o-4-[(2-o x o-5-o x a z o l i d i n y l)] m e t h o x y p h e n y l]-2- \\
\text { oxazolidinone }\end{array}$ & 29 & $\mathrm{~F}_{1}$ & {$[65]$} \\
\hline & $\begin{array}{l}\left(7 R^{*}, 11 S^{*}\right)-5-[3,5 \text {-Dibromo-4-[(2-oxo-5-oxazolidinyl)]methoxyphenyl]-2- } \\
\text { oxazolidinone }\end{array}$ & 30 & $\mathrm{~F}_{2}$ & {$[71]$} \\
\hline & 11,19-Dideoxyfistularin-3 & 33 & $\mathrm{G}_{1}$ & [104] \\
\hline & Aerothionin & 41 & $\mathrm{G}_{1}$ & {$[104]$} \\
\hline & Fistularin-3 & 46 & $\mathrm{G}_{2}$ & [104] \\
\hline & 11-Hydroxyaerothionin & 50 & $\mathrm{G}_{2}$ & {$[104]$} \\
\hline & 11-Oxoaerothionin & 52 & $\mathrm{G}_{2}$ & [104] \\
\hline \multirow{3}{*}{$\begin{array}{c}\text { A. laevis } \\
\text { (Carter, 1885) }\end{array}$} & $\begin{array}{l}\left(1^{\prime} R, 5^{\prime} R, 6 ' S\right)-2-\left(3^{\prime}, 5^{\prime}-\text { Dibromo-1',6'-dihydroxy-4'-oxo-cyclohex-2'-enyl) }\right. \\
\text { acetonitrile }\end{array}$ & 98 & $\mathrm{I}_{2}$ & {$[27]$} \\
\hline & $\begin{array}{l}\left(1^{\prime} R, 5^{\prime} S, 6^{\prime} S\right)-2-\left(3^{\prime}, 5^{\prime}-\text { Dibromo-1',6'-dihydroxy-4'-oxo-cyclohex-2'-enyl) }\right. \\
\text { acetonitrile }\end{array}$ & 99 & $\mathrm{I}_{2}$ & {$[27]$} \\
\hline & $(+)$ Aeroplysinin 1 & 100 & $\mathrm{~J}$ & {$[27]$} \\
\hline \multirow{10}{*}{ A. species } & Aplysamine1 & 1 & A & [84] \\
\hline & Aplysamine2 & 2 & A & {$[84]$} \\
\hline & Aplyzanzine A & 3 & A & {$[105]$} \\
\hline & $\begin{array}{l}\text { 2-(3,5-Dibromo-4-ethoxy-1-hydroxy-4-methoxy-2,5-cyclohexadien-1-yl)- } \\
\text { ethanamide }\end{array}$ & 22 & $\mathrm{D}_{2}$ & {$[71,84,106]$} \\
\hline & Aeroplysinine 2 & 25 & $\mathrm{E}_{1}$ & [106] \\
\hline & $\begin{array}{l}\left(7 R^{*}, 11 S^{*}\right)-5-[3,5-D i b r o m o-4-[(2-o x o-5-o x a z o l i d i n y l)] m e t h o x y p h e n y l]-2- \\
\text { oxazolidinone }\end{array}$ & 30 & $\mathrm{~F}_{2}$ & {$[71]$} \\
\hline & $(R, R)$-5[3,5-Dibromo-4-[(2-oxo-5-oxazolidinyl)]methoxyphenyl]-2-oxazolidone & 31 & $\mathrm{~F}_{1}$ & {$[106]$} \\
\hline & 11,19-Dideoxyfistularin-3 & 33 & $\mathrm{G}_{1}$ & {$[72]$} \\
\hline & 11-Oxofistularin-3 & 38 & $\mathrm{G}_{1}$ & {$[72]$} \\
\hline & 19-Deoxy-11-oxofistularin & 39 & $\mathrm{G}_{1}$ & {$[72]$} \\
\hline
\end{tabular}


Table 2. Cont

\begin{tabular}{|c|c|c|c|c|}
\hline \multirow{5}{*}{ A. species } & 19-Deoxyfistularin-3 & 40 & $\mathrm{G}_{1}$ & {$[72]$} \\
\hline & Aerothionin & 41 & $\mathrm{G}_{1}$ & [107] \\
\hline & Hemifistularin-3 & 71 & $\mathrm{G}_{7}$ & [72] \\
\hline & $\begin{array}{l}\text { (10R)-Ethyl-7,9-dibromo-10-hydroxy-8-methyl-1-oxa-2-azaspiro[4.5]deca- } \\
\text { 2,6,8-triene-3-carboxylate }\end{array}$ & 81 & $\mathrm{G}_{9}$ & [106] \\
\hline & 2-(3-Bromo-4-hydroxyphenyl)- $N, N, N$-trimethylethanaminium & 82 & $\mathrm{H}$ & [108] \\
\hline \multirow{11}{*}{$\begin{array}{c}\text { A. thiona } \\
\text { (Laubenfels, } \\
\text { 1950) }\end{array}$} & Aplysinketal A & 23 & $\mathrm{D}_{1}$ & [109] \\
\hline & Aplysinketal B & 24 & $\mathrm{D}_{1}$ & [109] \\
\hline & Aplysinolide & 27 & $\mathrm{E}_{2}$ & [109] \\
\hline & Aplysinimine & 28 & $\mathrm{E}_{2}$ & [109] \\
\hline & $\begin{array}{l}\left.\left.\left(7 R^{*}, 11 S^{*}\right)-5-[3,5 \text {-Dibromo-4-[(2-oxo-5-oxazolidinyl })\right]-m e t h o x y p h e n y l\right]-2- \\
\text { oxazolidinone }\end{array}$ & 30 & $\mathrm{~F}_{2}$ & [109] \\
\hline & Aerothionin & 41 & $\mathrm{G}_{1}$ & [109] \\
\hline & Homoaerothionin & 47 & $\mathrm{G}_{2}$ & [109] \\
\hline & 2-(3,5-Dibromo-2-hydroxy-4-methoxyphenyl)-acetamide & 84 & $\mathrm{H}$ & [109] \\
\hline & 2,6-Dibromo-4-acetamide-4-hydroxycyclohexadienone & 88 & $\mathrm{I}_{1}$ & [109] \\
\hline & Aplysina hydroxydienone or Dibromo compound 10 & 96 & $\mathrm{I}_{1}$ & [109] \\
\hline & Aplysina hydroxydienoic methyl esther & 97 & $\mathrm{I}_{1}$ & [109] \\
\hline
\end{tabular}


Table 3. Basic skeletons of the halogenated substances isolated from sponges of the Aplysina.

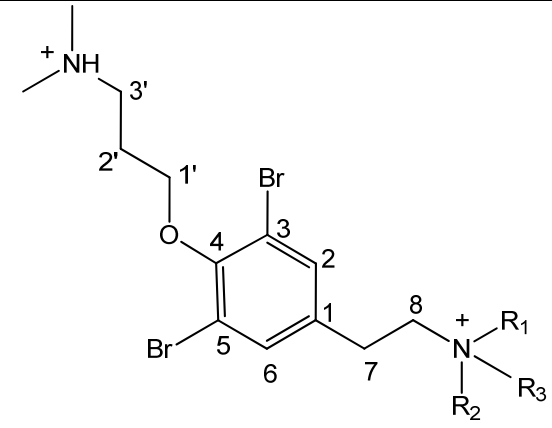

Code A

\section{Cavernicolins}

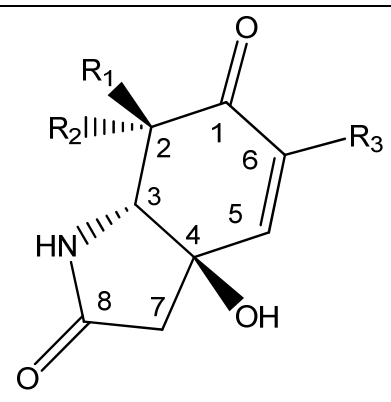

Code $\mathrm{B}_{1}$

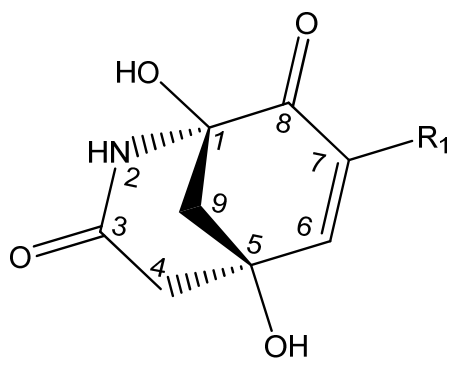

Code $\mathbf{B}_{3}$

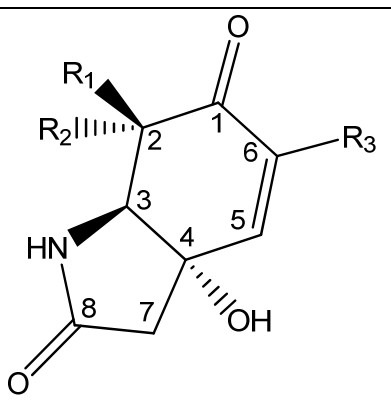

Code $\mathbf{B}_{2}$

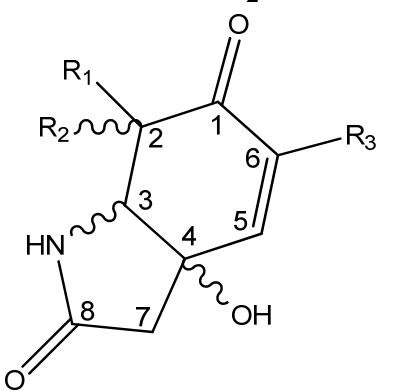

Code $\mathbf{B}_{4}$

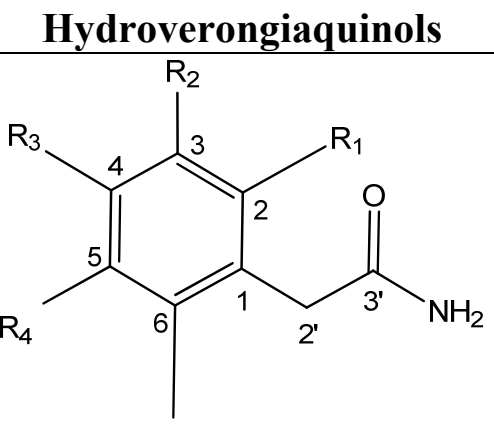

$\mathrm{R}_{5}$

Code C 
Table 3. Cont.

\section{Bromotyrosineketals}

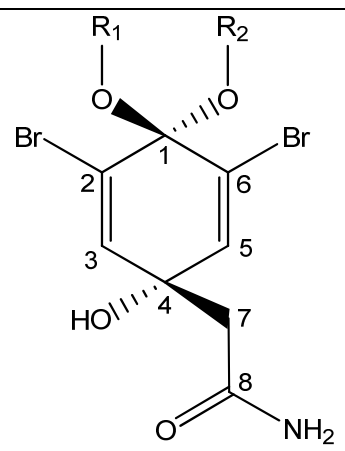

Code $D_{1}$

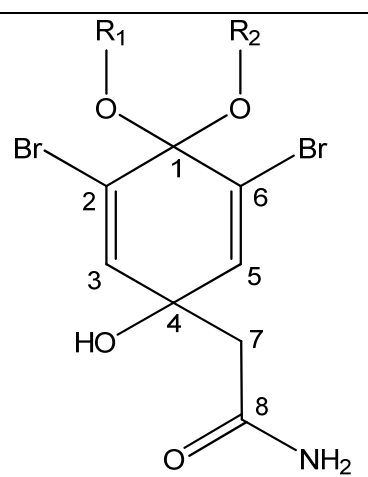

Code $\mathrm{D}_{2}$<smiles>[R1]C1=C(Br)C2([R])C(=CC1Br)OC(=O)C2[R]</smiles>

Code $\mathbf{E}_{1}$

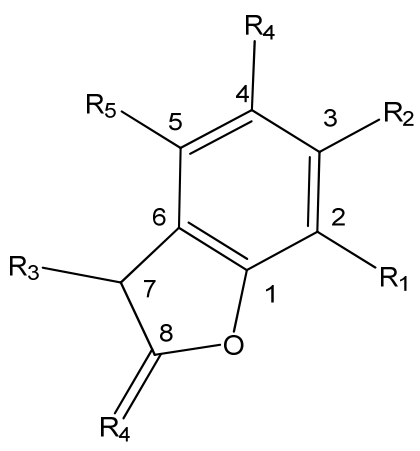

Code $\mathbf{E}_{2}$

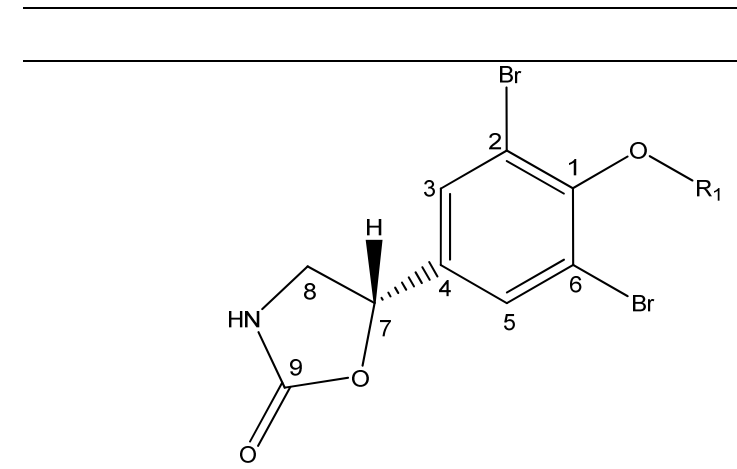

Code $\mathbf{F}_{1}$

\section{Oxazolidones}

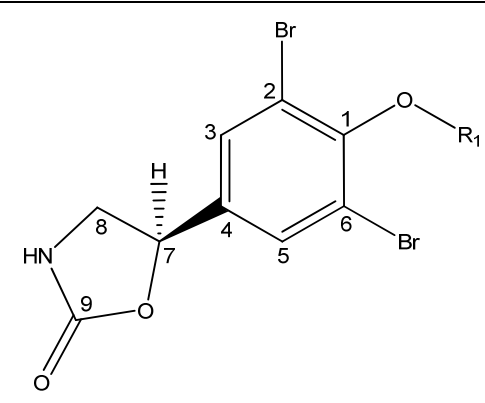

Code $\mathbf{F}_{2}$

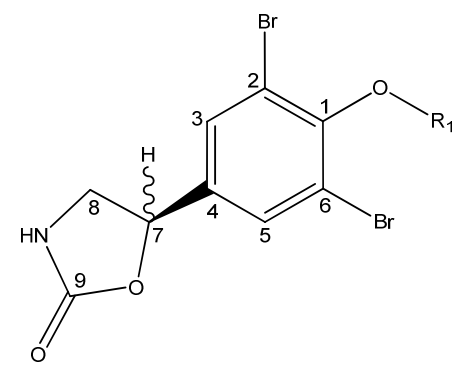

Code $\mathbf{F}_{3}$ 
Table 3. Cont.

\section{Spiroisoxazolines}

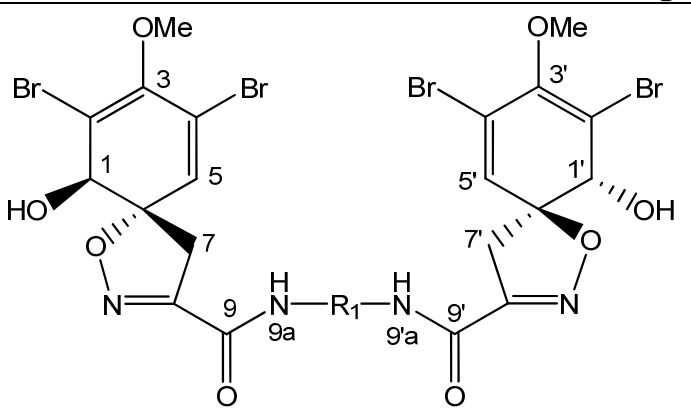

Code $\mathbf{G}_{\mathbf{1}}$

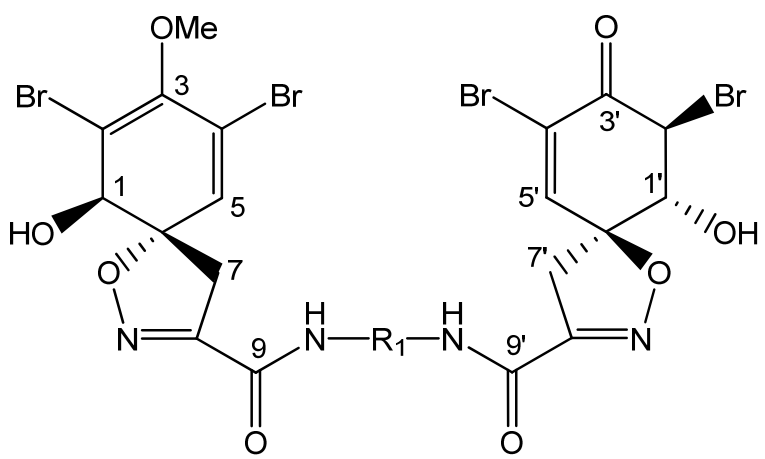

Code $\mathbf{G}_{\mathbf{3}}$

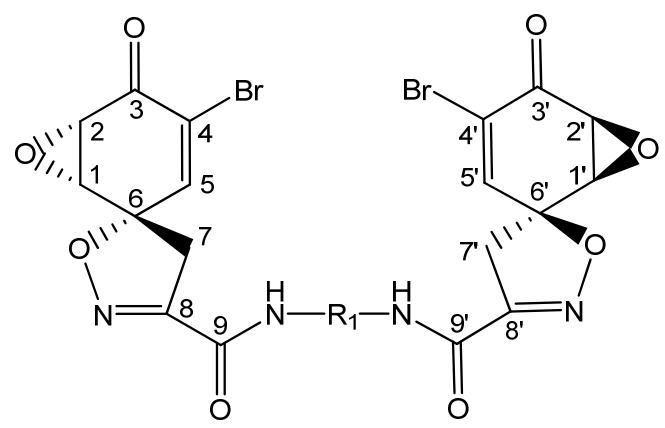

Code $\mathbf{G}_{5}$

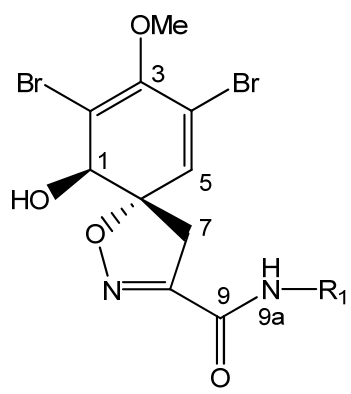

Code $\mathbf{G}_{7}$

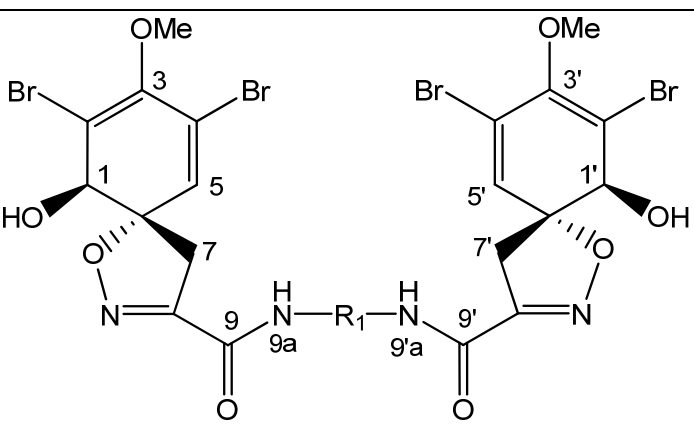

Code $\mathbf{G}_{\mathbf{2}}$

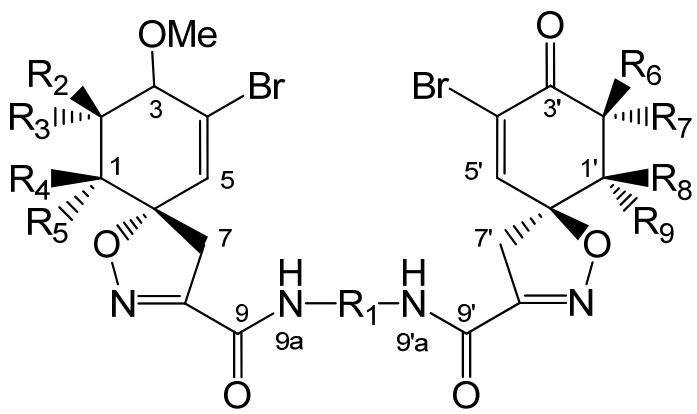

Code $\mathbf{G}_{4}$

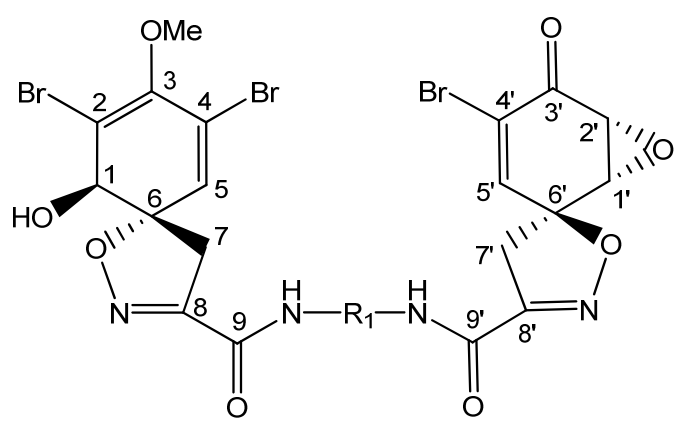

Code $\mathbf{G}_{6}$

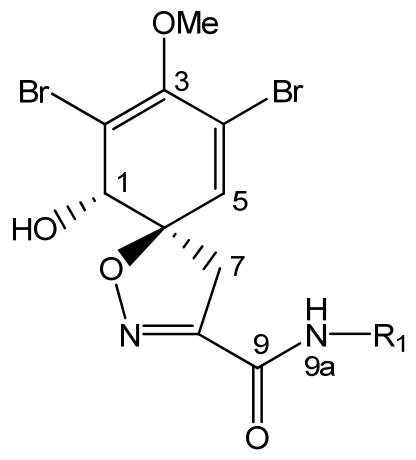

Code $\mathbf{G}_{8}$ 
Table 3. Cont.

\section{Spiroisoxazolines}

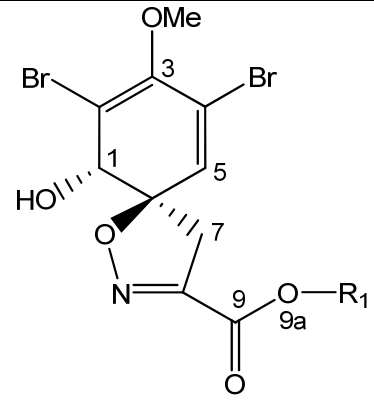

Code G9

Verongiabenzenoids<smiles>[R]c1cc(Br)c(OC)c(Br)c1[R]</smiles>

Code H

\section{Verongiaquinols}

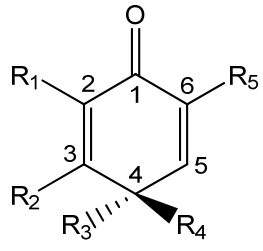

Code $I_{1}$

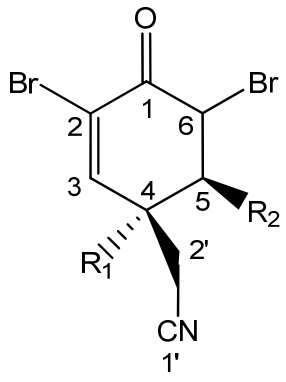

Code $\mathrm{I}_{2}$

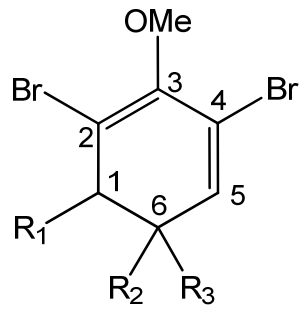

\section{Code J}


Table 4. Substituent groups of the halogenated substances.

(E) $\mathrm{CH}_{2}=\mathrm{CH}_{2}$


Table 4. Cont.

Spiroisoxazolines


Table 4. Cont.

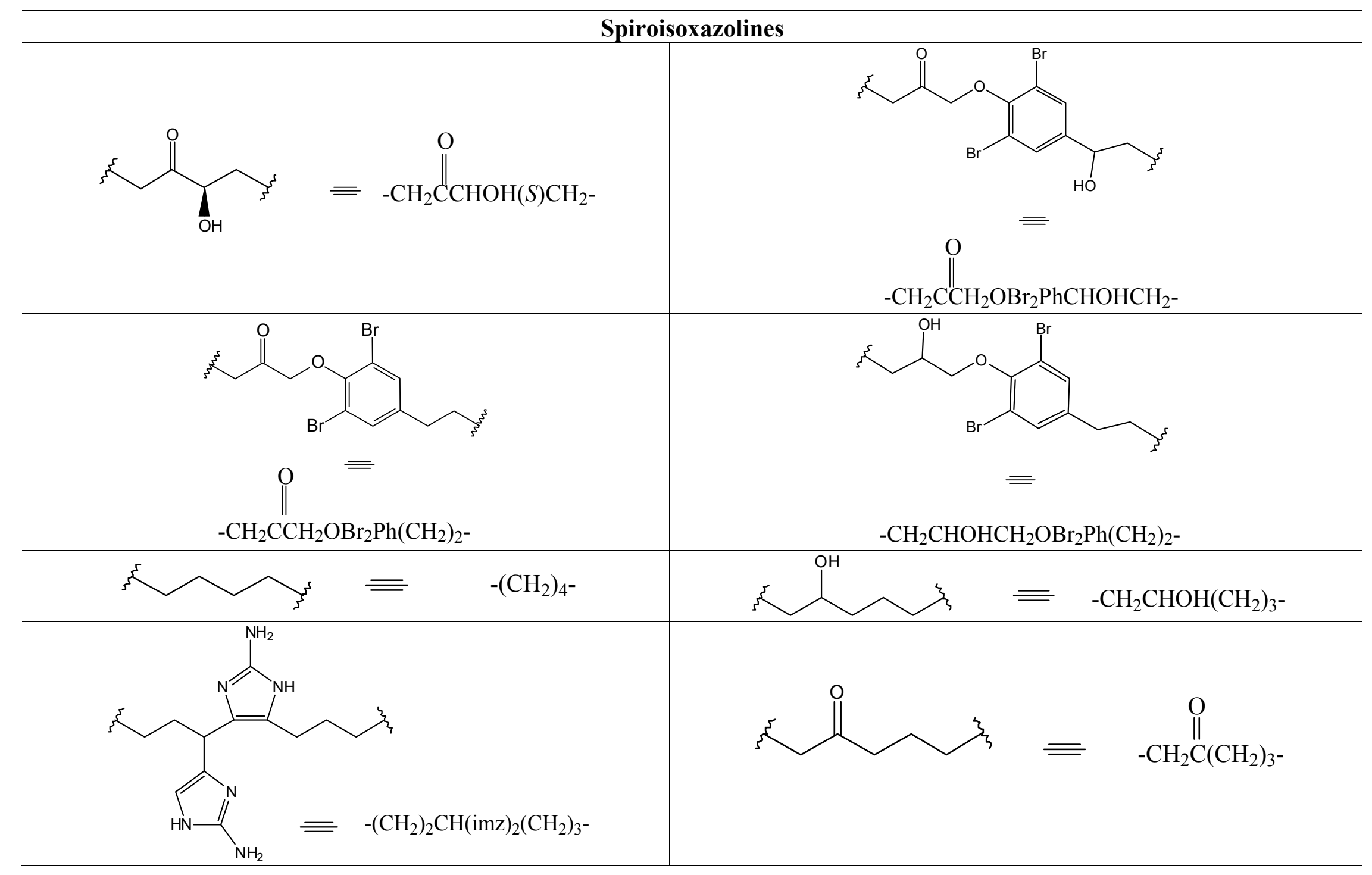


Table 4. Cont.

(1)


Table 4. Cont.

(n)


Table 4. Cont.

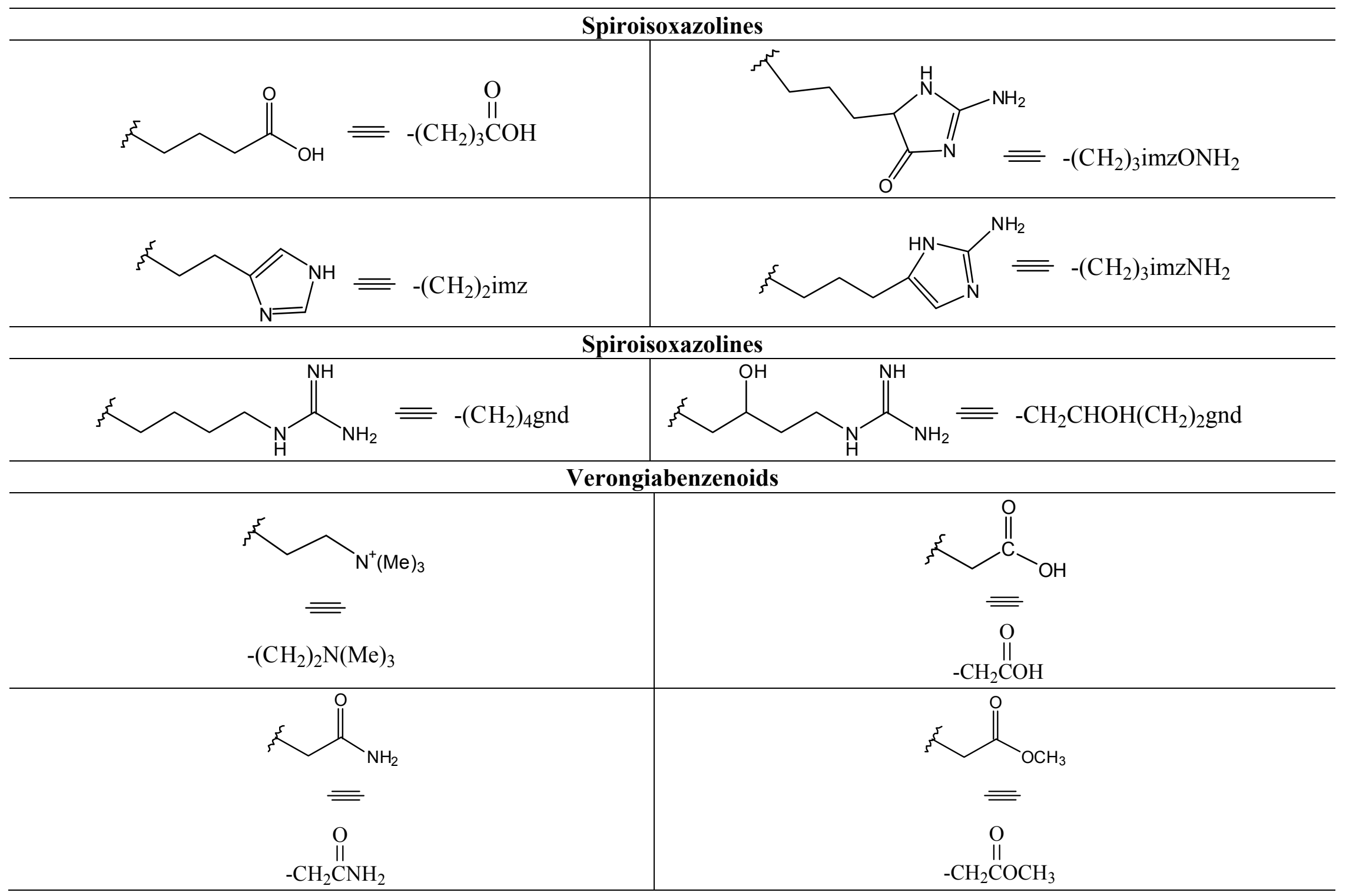


Table 4. Cont.

$\overline{0}$ Verongiaquinols

Table 5. Halogenated substances found in the genus Aplysina.

\begin{tabular}{|c|c|c|c|c|c|c|c|c|c|c|}
\hline $\begin{array}{c}\text { Halogen } \\
\text { Compound }\end{array}$ & $\mathbf{R}^{1}$ & $\mathbf{R}^{2}$ & $\mathbf{R}^{3}$ & $\mathbf{R}^{4}$ & $\mathbf{R}^{5}$ & $\mathbf{R}^{6}$ & $\mathbf{R}^{7}$ & $\mathbf{R}^{8}$ & $\mathbf{R}^{9}$ & Nucleus \\
\hline 1 & $\mathrm{Me}$ & $\mathrm{Me}$ & - & - & - & - & - & - & - & $\mathrm{A}$ \\
\hline 2 & $\mathrm{H}$ & (E) $\mathrm{BrMeOPhCH}_{2} \mathrm{CNOHCO}-$ & - & - & - & - & - & - & - & $\mathrm{A}$ \\
\hline 3 & $\mathrm{H}$ & $\mathrm{Br}_{2} \mathrm{MeOPhCH}{ }_{2} \mathrm{CHN}\left(\mathrm{CH}_{3}\right)_{2} \mathrm{CO}-$ & - & - & - & - & - & - & - & A \\
\hline 4 & $\mathrm{Me}$ & $\mathrm{Me}$ & $\mathrm{Me}$ & - & - & - & - & - & - & $\mathrm{A}$ \\
\hline 5 & $\mathrm{Br}$ & $\mathrm{H}$ & $\mathrm{Br}$ & - & - & - & - & - & - & $\mathrm{B}_{1}$ \\
\hline 6 & $\mathrm{H}$ & $\mathrm{Br}$ & $\mathrm{Br}$ & - & - & - & - & - & - & $\mathrm{B}_{1}$ \\
\hline 7 & $\mathrm{Cl}$ & $\mathrm{H}$ & $\mathrm{Br}$ & - & - & - & - & - & - & $\mathrm{B}_{2}$ \\
\hline 8 & $\mathrm{H}$ & $\mathrm{Cl}$ & $\mathrm{Br}$ & - & - & - & - & - & - & $\mathrm{B}_{2}$ \\
\hline 9 & $\mathrm{Br}$ & $\mathrm{H}$ & $\mathrm{Cl}$ & - & - & - & - & - & - & $\mathrm{B}_{2}$ \\
\hline 10 & $\mathrm{H}$ & $\mathrm{Br}$ & $\mathrm{Cl}$ & - & - & - & - & - & - & $\mathrm{B}_{2}$ \\
\hline 11 & $\mathrm{H}$ & $\mathrm{H}$ & $\mathrm{Br}$ & - & - & - & - & - & - & $\mathrm{B}_{2}$ \\
\hline 12 & $\mathrm{H}$ & $\mathrm{H}$ & $\mathrm{Cl}$ & - & - & - & - & - & - & $\mathrm{B}_{2}$ \\
\hline 13 & $\mathrm{Br}$ & - & - & - & - & - & - & - & - & $\mathrm{B}_{3}$ \\
\hline 14 & $\mathrm{Cl}$ & - & - & - & - & - & - & - & - & $\mathrm{B}_{3}$ \\
\hline 15 & $\mathrm{H}$ & $\mathrm{Cl}$ & $\mathrm{Cl}$ & - & - & - & - & - & - & $\mathrm{B}_{4}$ \\
\hline 16 & $\mathrm{H}$ & $\mathrm{Cl}$ & $\mathrm{Br}$ & - & - & - & - & - & - & $\mathrm{B}_{4}$ \\
\hline 17 & $\mathrm{H}$ & $\mathrm{Br}$ & $\mathrm{Br}$ & - & - & - & - & - & - & $\mathrm{B}_{4}$ \\
\hline 18 & $\mathrm{OH}$ & $\mathrm{H}$ & $\mathrm{Br}$ & $\mathrm{OH}$ & $\mathrm{Br}$ & - & - & - & - & $\mathrm{C}$ \\
\hline 19 & $\mathrm{Br}$ & $\mathrm{OH}$ & $\mathrm{Br}$ & $\mathrm{H}$ & $\mathrm{OH}$ & - & - & - & - & $\mathrm{C}$ \\
\hline 20 & $\mathrm{H}$ & $\mathrm{Br}$ & $\mathrm{OH}$ & $\mathrm{Br}$ & $\mathrm{H}$ & - & - & - & - & $\mathrm{C}$ \\
\hline 21 & $\mathrm{Me}$ & $\mathrm{Me}$ & - & - & - & - & - & - & - & $\mathrm{D}_{1}$ \\
\hline 22 & $\mathrm{Et}$ & $\mathrm{Me}$ & - & - & - & - & - & - & - & $\mathrm{D}_{2}$ \\
\hline 23 & $\mathrm{Me}$ & Butyl & - & - & - & - & - & - & - & $\mathrm{D}_{1}$ \\
\hline 24 & $\mathrm{Me}$ & Pentyl & - & - & - & - & - & - & - & $\mathrm{D}_{1}$ \\
\hline 25 & $\mathrm{MeO}$ & $\mathrm{OH}$ & $\mathrm{H}$ & - & - & - & - & - & - & $\mathrm{E}_{1}$ \\
\hline
\end{tabular}


Table 5. Cont.

\begin{tabular}{|c|c|c|c|c|c|c|c|c|c|c|}
\hline 26 & $\mathrm{H}$ & $\mathrm{Br}$ & (E) $\mathrm{CH}_{2}=\mathrm{CH}=\mathrm{CH}=\mathrm{CH}-$ & $\mathrm{OH}$ & $\mathrm{Br}$ & - & - & - & - & $\mathrm{E}_{1}$ \\
\hline 27 & $\mathrm{Br}$ & $\mathrm{OMe}$ & $\underset{\mathrm{CH}_{3} \mathrm{CCH}_{3}}{\|}$ & $\mathrm{Br}$ & $\mathrm{H}$ & - & - & - & - & $\mathrm{E}_{2}$ \\
\hline 28 & $\mathrm{Br}$ & $\mathrm{OMe}$ & $\mathrm{H}$ & $\mathrm{Br}$ & $\mathrm{H}$ & - & - & - & - & $\mathrm{E}_{2}$ \\
\hline 29 & $-\mathrm{CH}_{2}(R) \mathrm{oxz}$ & - & - & - & - & - & - & - & - & $\mathrm{F}_{1}$ \\
\hline 30 & $-\mathrm{CH}_{2}(\mathrm{~S}) \mathrm{oxz}$ & - & - & - & - & - & - & - & - & $\mathrm{F}_{2}$ \\
\hline 31 & $-\mathrm{CH}_{2}(R) \mathrm{oxz}$ & - & - & - & - & - & - & - & - & $\mathrm{F}_{1}$ \\
\hline 32 & $-\mathrm{CH}_{2} \mathrm{MeOoxz}$ & - & - & - & - & - & - & - & - & $\mathrm{F}_{3}$ \\
\hline 33 & $-\left(\mathrm{CH}_{2}\right)_{3} \mathrm{OBr}_{2} \mathrm{Ph}\left(\mathrm{CH}_{2}\right)_{2^{-}}$ & - & - & - & - & - & - & - & - & $\mathrm{G}_{1}$ \\
\hline 34 & $-\mathrm{CH}_{2}(\mathrm{R}) \mathrm{CHOHCH}_{2} \mathrm{OBr}_{2} \mathrm{PhCHOHCH}_{2-}$ & - & - & - & - & - & - & - & - & $\mathrm{G}_{1}$ \\
\hline 35 & $-\left(\mathrm{CH}_{2}\right)_{3} \mathrm{OBr}_{2} \mathrm{Ph}(R) \mathrm{CHOHCH}_{2^{-}}$ & - & - & - & - & - & - & - & - & $\mathrm{G}_{1}$ \\
\hline 36 & $\begin{array}{c}\mathrm{O} \\
\stackrel{\mathrm{CH}_{2} \mathrm{CCHOH}(R) \mathrm{CH}_{2^{-}}}{\mathrm{C}} \\
\end{array}$ & - & - & - & - & - & - & - & - & $\mathrm{G}_{1}$ \\
\hline 37 & 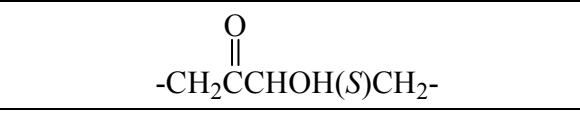 & - & - & - & - & - & - & - & - & $\mathrm{G}_{1}$ \\
\hline 38 & $\stackrel{\mathrm{O}}{\|}-\mathrm{CH}_{2} \mathrm{CCH}_{2} \mathrm{OBr}_{2} \mathrm{PhCHOHCH}_{2}-$ & - & - & - & - & - & - & - & - & $\mathrm{G}_{1}$ \\
\hline 39 & $\begin{array}{l}\mathrm{O} \\
\| \\
-\mathrm{CH}_{2} \mathrm{CCH}_{2} \mathrm{OBr}_{2} \mathrm{Ph}\left(\mathrm{CH}_{2}\right)_{2}^{-}\end{array}$ & - & - & - & - & - & - & - & - & $\mathrm{G}_{1}$ \\
\hline 40 & $-\mathrm{CH}_{2} \mathrm{CHOHCH}_{2} \mathrm{OBr}_{2} \mathrm{Ph}\left(\mathrm{CH}_{2}\right)_{2-}$ & - & - & - & - & - & - & - & - & $\mathrm{G}_{1}$ \\
\hline 41 & $-\left(\mathrm{CH}_{2}\right)_{4^{-}}$ & - & - & - & - & - & - & - & - & $\mathrm{G}_{1}$ \\
\hline 42 & $-\mathrm{CH}_{2} \mathrm{CHOH}\left(\mathrm{CH}_{2}\right)_{3^{-}}$ & - & - & - & - & - & - & - & - & $\mathrm{G}_{1}$ \\
\hline 43 & $-\left(\mathrm{CH}_{2}\right)_{2} \mathrm{CH}(\mathrm{imz})_{2}\left(\mathrm{CH}_{2}\right)_{3^{-}}$ & - & - & - & - & - & - & - & - & $\mathrm{G}_{1}$ \\
\hline 44 & $\begin{array}{c}\stackrel{\mathrm{O}}{\|} \\
-\mathrm{CH}_{2} \mathrm{C}\left(\mathrm{CH}_{2}\right)_{3-} \\
\end{array}$ & - & - & - & - & - & - & - & - & $\mathrm{G}_{1}$ \\
\hline 45 & $-\left(\mathrm{CH}_{2}\right)_{3} \mathrm{OBr}_{2} \mathrm{PhCHOHCH}_{2-}$ & - & - & - & - & - & - & - & - & $\mathrm{G}_{2}$ \\
\hline
\end{tabular}


Table 5. Cont

\begin{tabular}{|c|c|c|c|c|c|c|c|c|c|c|}
\hline 46 & $-\mathrm{CH}_{2}(S) \mathrm{CHOHCH}_{2} \mathrm{OBr}_{2} \mathrm{PhCHOHCH}_{2}-$ & - & - & - & - & - & - & - & - & $\mathrm{G}_{2}$ \\
\hline 47 & $-\left(\mathrm{CH}_{2}\right)_{5^{-}}$ & - & - & - & - & - & - & - & - & $\mathrm{G}_{2}$ \\
\hline 48 & $-\mathrm{CH}_{2} \mathrm{CHOHCH}_{2} \mathrm{OBr}_{2} \mathrm{PhCHOHCH}_{2-}^{-}$ & - & - & - & - & - & - & - & - & $\mathrm{G}_{2}$ \\
\hline 49 & $-\mathrm{CH}_{2}(\mathrm{CHOH})_{2} \mathrm{CH}_{2^{-}}$ & - & - & - & - & - & - & - & - & $\mathrm{G}_{2}$ \\
\hline 50 & $-\mathrm{CH}_{2} \mathrm{CHOH}(\mathrm{CH})_{2-}^{-}$ & - & - & - & - & - & - & - & - & $\mathrm{G}_{2}$ \\
\hline 51 & $\stackrel{\mathrm{O}}{-\mathrm{CH}_{2} \mathrm{CCH}_{2} \mathrm{OBr}_{2} \mathrm{PhCHOHCH}_{2-}-}$ & - & - & - & - & - & - & - & - & $\mathrm{G}_{2}$ \\
\hline 52 & $-\mathrm{CH}_{2} \mathrm{CH}_{2} \mathrm{O}\left(\mathrm{CH}_{2}\right)_{2}^{-}$ & - & - & - & - & - & - & - & - & $\mathrm{G}_{2}$ \\
\hline 53 & $-\left(\mathrm{CH}_{2}\right)_{5} \mathrm{COHCH}_{2}^{-}$ & - & - & - & - & - & - & - & - & $\mathrm{G}_{2}$ \\
\hline 54 & $-\left(\mathrm{CH}_{2}\right)_{5^{-}}$ & - & - & - & - & - & - & - & - & $\mathrm{G}_{3}$ \\
\hline 55 & $-\left(\mathrm{CH}_{2}\right)_{4^{-}}$ & - & - & - & - & - & - & - & - & $\mathrm{G}_{3}$ \\
\hline 56 & $-\left(\mathrm{CH}_{2}\right)_{5-}^{-}$ & $\mathrm{H}$ & $\mathrm{Br}$ & $\mathrm{OH}$ & $\mathrm{H}$ & $\mathrm{Br}$ & $\mathrm{H}$ & $\mathrm{H}$ & $\mathrm{OH}$ & $\mathrm{G}_{4}$ \\
\hline 57 & $-\mathrm{CH}_{2} \mathrm{CH}_{2} \mathrm{O}\left(\mathrm{CH}_{2}\right)_{2-}$ & $\mathrm{Br}$ & $\mathrm{H}$ & $\mathrm{H}$ & $\mathrm{OH}$ & $\mathrm{H}$ & $\mathrm{Br}$ & $\mathrm{OH}$ & $\mathrm{H}$ & $\mathrm{G}_{4}$ \\
\hline 58 & $-\mathrm{CH}_{2} \mathrm{CHOH}(\mathrm{CH})_{2-}^{-}$ & $\mathrm{H}$ & $\mathrm{Br}$ & $\mathrm{OH}$ & $\mathrm{H}$ & $\mathrm{Br}$ & $\mathrm{H}$ & $\mathrm{H}$ & $\mathrm{OH}$ & $\mathrm{G}_{4}$ \\
\hline 59 & $-\mathrm{CH}_{2} \mathrm{CHOH}(\mathrm{CH})_{2^{-}}$ & $\mathrm{Br}$ & $\mathrm{H}$ & $\mathrm{OH}$ & $\mathrm{H}$ & $\mathrm{H}$ & $\mathrm{Br}$ & $\mathrm{H}$ & $\mathrm{OH}$ & $\mathrm{G}_{4}$ \\
\hline 60 & $-\mathrm{CH}_{2} \mathrm{CHOH}\left(\mathrm{CH}_{2}\right)_{3^{-}}$ & $\mathrm{H}$ & $\mathrm{Br}$ & $\mathrm{OH}$ & $\mathrm{H}$ & $\mathrm{Br}$ & $\mathrm{H}$ & $\mathrm{H}$ & $\mathrm{OH}$ & $\mathrm{G}_{4}$ \\
\hline 61 & $-\mathrm{CH}_{2} \mathrm{CHOH}\left(\mathrm{CH}_{2}\right)_{3-}^{-}$ & $\mathrm{Br}$ & $\mathrm{H}$ & $\mathrm{OH}$ & $\mathrm{H}$ & $\mathrm{H}$ & $\mathrm{Br}$ & $\mathrm{H}$ & $\mathrm{OH}$ & $\mathrm{G}_{4}$ \\
\hline 62 & $-\left(\mathrm{CH}_{2}\right)_{4^{-}}$ & - & - & - & - & - & - & - & - & $\mathrm{G}_{5}$ \\
\hline 63 & $-\left(\mathrm{CH}_{2}\right)_{5^{-}}$ & - & - & - & - & - & - & - & - & $\mathrm{G}_{6}$ \\
\hline 64 & $\begin{array}{cc}\mathrm{O} & \mathrm{O} \\
\| & \| \\
-\left(\mathrm{CH}_{2}\right)_{2} \mathrm{CCH}_{2} \mathrm{NHCOCH}_{3} & \end{array}$ & - & - & - & - & - & - & - & - & $\mathrm{G}_{7}$ \\
\hline 65 & $\begin{array}{cc}\mathrm{O} & \mathrm{O} \\
\| & \| \\
-\mathrm{CH}_{2} \mathrm{C}\left(\mathrm{CH}_{2}\right)_{2} \mathrm{NHCOMe} & \\
\end{array}$ & - & - & - & - & - & - & - & - & $\mathrm{G}_{7}$ \\
\hline 66 & $\begin{array}{c}\stackrel{\mathrm{O}}{\|} \\
-\left(\mathrm{CH}_{2}\right)_{4} \mathrm{NHCOMe} \\
\end{array}$ & - & - & - & - & - & - & - & - & $\mathrm{G}_{7}$ \\
\hline 67 & $-\mathrm{CH}_{2}(\mathrm{Z})(\mathrm{CH})_{2} \mathrm{imzNH}_{2}$ & - & - & - & - & - & - & - & - & $\mathrm{G}_{7}$ \\
\hline 68 & $-\left(\mathrm{CH}_{2}\right)_{5}$ gnd & - & - & - & - & - & - & - & - & $\mathrm{G}_{7}$ \\
\hline 69 & $-\left(\mathrm{CH}_{2}\right)_{3} \mathrm{OBr}_{2} \mathrm{PhCHOHCH}_{2} \mathrm{NHAc}$ & - & - & - & - & - & - & - & - & $\mathrm{G}_{7}$ \\
\hline 70 & $-\left(\mathrm{CH}_{2}\right)_{3} \mathrm{OBr}_{2} \mathrm{Ph}\left(\mathrm{CH}_{2}\right)_{2} \mathrm{NHSO}_{3} \mathrm{Na}$ & - & - & - & - & - & - & - & - & $\mathrm{G}_{7}$ \\
\hline
\end{tabular}


Table 5. Cont.

\begin{tabular}{|c|c|c|c|c|c|c|c|c|c|c|}
\hline 71 & $-\mathrm{CH}_{2} \mathrm{CHOHBr}_{2} \mathrm{PhOH}$ & - & - & - & - & - & - & - & - & $\mathrm{G}_{7}$ \\
\hline 72 & $-\left(\mathrm{CH}_{2}\right)_{3} \mathrm{OBr}_{2} \mathrm{Phoxz}$ & - & - & - & - & - & - & - & - & $\mathrm{G}_{7}$ \\
\hline 73 & $-\mathrm{CH}_{2} \mathrm{Br}_{2} \mathrm{PhOoxz}$ & - & - & - & - & - & - & - & - & $\mathrm{G}_{7}$ \\
\hline 74 & $\begin{array}{c}\mathrm{O} \\
\stackrel{\mathrm{I}}{\mathrm{COH}} \\
-\left(\mathrm{CH}_{2}\right)_{3} \mathrm{COH} \\
\end{array}$ & - & - & - & - & - & - & - & - & $\mathrm{G}_{7}$ \\
\hline 75 & $-\left(\mathrm{CH}_{2}\right)_{3} \mathrm{imzONH}_{2}$ & - & - & - & - & - & - & - & - & $\mathrm{G}_{7}$ \\
\hline 76 & $-\left(\mathrm{CH}_{2}\right)_{2} \mathrm{imz}$ & - & - & - & - & - & - & - & - & $\mathrm{G}_{7}$ \\
\hline 77 & $-\left(\mathrm{CH}_{2}\right)_{3} \mathrm{imzNH}_{2}$ & - & - & - & - & - & - & - & - & $\mathrm{G}_{7}$ \\
\hline 78 & $-\left(\mathrm{CH}_{2}\right)_{4}$ gnd & - & - & - & - & - & - & - & - & $\mathrm{G}_{7}$ \\
\hline 79 & $-\mathrm{CH}_{2} \mathrm{CHOH}\left(\mathrm{CH}_{2}\right)_{2}$ gnd & - & - & - & - & - & - & - & - & $\mathrm{G}_{8}$ \\
\hline 80 & $\mathrm{Me}$ & - & - & - & - & - & - & - & - & $\mathrm{G}_{9}$ \\
\hline 81 & $\mathrm{Et}$ & - & - & - & - & - & - & - & - & $\mathrm{G}_{9}$ \\
\hline 82 & $-\left(\mathrm{CH}_{2}\right)_{2} \mathrm{~N}(\mathrm{Me})_{3}$ & $\mathrm{H}$ & - & - & - & - & - & - & - & $\mathrm{H}$ \\
\hline 83 & $\begin{array}{c}\stackrel{\mathrm{O}}{\|} \\
-\mathrm{CH}_{2} \mathrm{COH} \\
\end{array}$ & $\mathrm{OH}$ & - & - & - & - & - & - & - & $\mathrm{H}$ \\
\hline 84 & $\begin{array}{c}\mathrm{O} \\
\| \\
-\mathrm{CH}_{2} \mathrm{CNH}_{2}\end{array}$ & $\mathrm{OH}$ & - & - & - & - & - & - & - & $\mathrm{H}$ \\
\hline 85 & $\mathrm{OH}$ & $\mathrm{H}$ & - & - & - & - & - & - & - & $\mathrm{H}$ \\
\hline 86 & $\begin{array}{c}\mathrm{O} \\
\stackrel{\|}{1} \\
-\mathrm{CH}_{2} \mathrm{COCH}_{3}\end{array}$ & $\mathrm{OH}$ & - & - & - & - & - & - & - & $\mathrm{H}$ \\
\hline 87 & $\mathrm{Br}$ & $\mathrm{H}$ & $\mathrm{H}$ & $\mathrm{O}$ & $\mathrm{Br}$ & - & - & - & - & $\mathrm{I}_{1}$ \\
\hline 88 & $\mathrm{Br}$ & $\mathrm{H}$ & $\mathrm{OH}$ & $\begin{array}{c}\mathrm{O} \\
\| \\
-\mathrm{CH}_{2} \mathrm{CNH}_{2}\end{array}$ & $\mathrm{Br}$ & - & - & - & - & $\mathrm{I}_{1}$ \\
\hline 89 & $\mathrm{Cl}$ & $\mathrm{H}$ & $\mathrm{OH}$ & $\begin{array}{c}\mathrm{O} \\
\stackrel{\|}{1} \\
-\mathrm{CH}_{2} \mathrm{CNH}_{2} \\
\end{array}$ & $\mathrm{Cl}$ & - & - & - & - & $\mathrm{I}_{1}$ \\
\hline 90 & $\mathrm{Br}$ & $\mathrm{H}$ & $\mathrm{OH}$ & $\begin{array}{c}\mathrm{O} \\
\stackrel{\|}{\|} \\
-\mathrm{CH}_{2} \mathrm{CNH}_{2} \\
\end{array}$ & $\mathrm{Cl}$ & - & - & - & - & $\mathrm{I}_{1}$ \\
\hline
\end{tabular}


Table 5. Cont

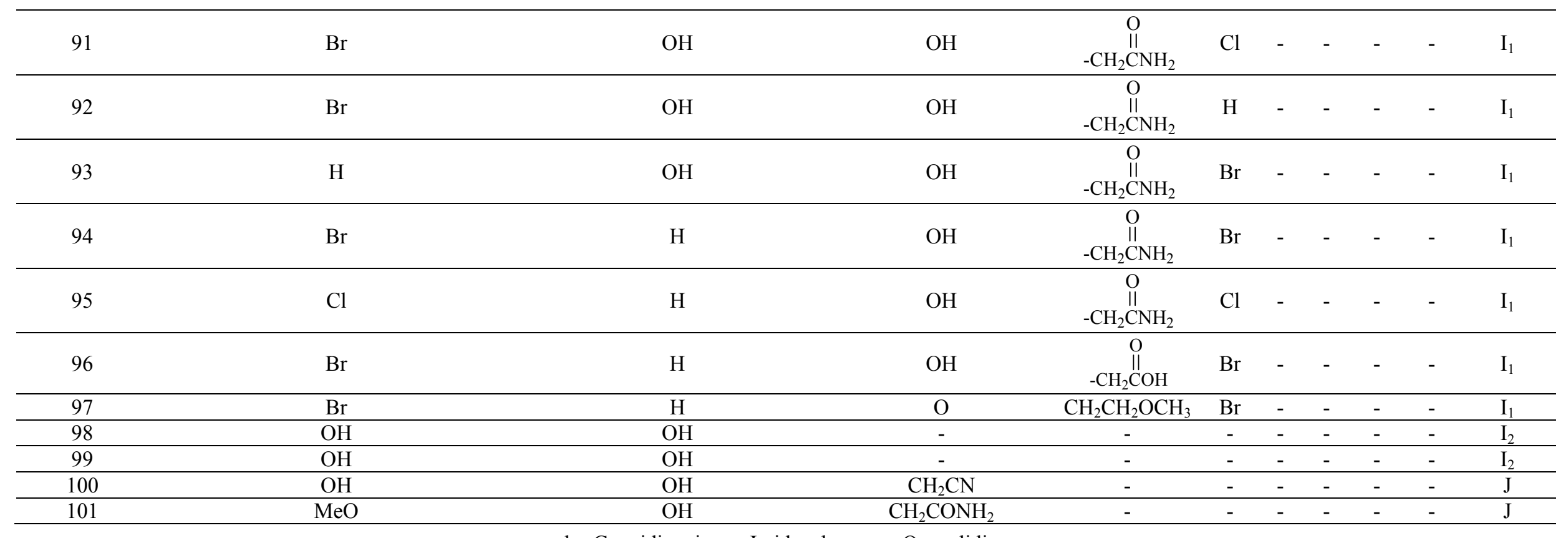

gnd $=$ Guanidine; imz = Imidazole; oxz = Oxazolidinone. 
Table 6. Compilation of the ${ }^{13} \mathrm{C}$ NMR data of the halogenated substances from the genus Aplysina. Bromotyramines.

\begin{tabular}{ccccc}
\hline Position & $\mathbf{1}$ & $\mathbf{2}$ & $\mathbf{3}$ & $\mathbf{4}$ \\
\hline 1 & 140.3 & 140.3 & 137.94 & 138.58 \\
2 & 134.4 & 134.4 & 132.78 & 132.80 \\
3 & 118.7 & 118.7 & 117.74 & 118.02 \\
4 & 152.1 & 152.1 & 150.87 & 151.30 \\
5 & 118.7 & 118.7 & 117.74 & 118.02 \\
6 & 134.4 & 134.4 & 132.78 & 132.80 \\
7 & 35.2 & 35.2 & 34.20 & 32.26 \\
8 & 41.3 & 41.3 & 39.81 & 60.20 \\
9 & - & 165.8 & 170.82 & - \\
10 & - & 152.9 & 69.84 & - \\
11 & - & 28.7 & 31.57 & - \\
12 & - & 113.1 & 137.66 & - \\
13 & - & 134.7 & 133.21 & - \\
14 & - & 130.3 & 117.58 & - \\
15 & - & 155.8 & 152.34 & - \\
16 & - & 112.1 & 117.58 & - \\
17 & - & 131.7 & 133.21 & - \\
1 & 71.7 & 71.7 & 69.71 & 71.00 \\
2 & 26.4 & 26.4 & 25.38 & 26.79 \\
$3{ }^{\prime}$ & 56.9 & 56.9 & 55.41 & 55.77 \\
$\mathrm{MeO}$ & - & 56.7 & 60.39 & - \\
${ }^{\mathrm{N}} \mathrm{N}(\mathrm{Me})_{2}$ & $43.7 / 43.6$ & 43.7 & $41.51 / 42.92$ & 44.06 \\
${ }^{\mathrm{N}} \mathrm{N}(\mathrm{Me})_{3}$ & - & - & - & 44.74 \\
\hline
\end{tabular}

Table 7. Compilation of the ${ }^{13} \mathrm{C}$ NMR data of the halogenated substances from the genus Aplysina. Cavernicolins.

\begin{tabular}{cccccccc}
\hline Position & $\mathbf{5}$ & $\mathbf{6}$ & $\mathbf{9}$ & $\mathbf{1 1}$ & $\mathbf{1 2}$ & $\mathbf{1 3}$ & $\mathbf{1 4}$ \\
\hline 1 & 184.0 & 184.0 & 183.4 & 188.91 & 188.89 & 80.90 & 82.7 \\
2 & 56.9 & 53.1 & 58.0 & 39.78 & 40.08 & - & - \\
3 & 68.7 & 64.7 & 67.9 & 58.60 & 58.56 & 168.30 & 171.4 \\
4 & 76.6 & 75.7 & 74.4 & 74.15 & 73.41 & 43.1 & 44.0 \\
5 & 149.9 & 149.0 & 146.3 & 150.07 & 145.84 & 69.5 & 69.5 \\
6 & 120.8 & 120.8 & 127.4 & 122.45 & 130.18 & 156.90 & 153.2 \\
7 & 43.0 & 45.1 & 42.4 & 43.58 & 43.78 & 118.80 & 129.5 \\
8 & 173.9 & 173.9 & 173.6 & 172.65 & 172.72 & 187.8 & 188.9 \\
9 & - & - & - & - & - & 46.6 & 47.6 \\
\hline
\end{tabular}


Table 8. Compilation of the ${ }^{13} \mathrm{C}$ NMR data of the halogenated substances from the genus Aplysina. Hydroverongiaquinols.

\begin{tabular}{cc}
\hline Position & $\mathbf{2 0}$ \\
\hline 1 & 130.0 \\
2 & 133.0 \\
3 & 111.0 \\
4 & 150.0 \\
5 & 111.0 \\
6 & 133.0 \\
2, & 40.0 \\
3, & 173.4 \\
\hline
\end{tabular}

Table 9. Compilation of the ${ }^{13} \mathrm{C}$ NMR data of the halogenated substances from the genus Aplysina. Bromotyrosineketals.

\begin{tabular}{ccc}
\hline Position & $\mathbf{2 2}$ & $\mathbf{2 3}$ \\
\hline 1 & 71.3 & 123.55 \\
2 & 140.3 & 123.55 \\
3 & 124.1 & 142.11 \\
4 & 96.7 & 71.92 \\
5 & 124.1 & 142.11 \\
6 & 140.3 & 123.55 \\
7 & 44.2 & 45.03 \\
8 & 173.2 & 172.99 \\
9 & 51.3 & 51.04 \\
10 & 60.2 & 63.98 \\
11 & 15.4 & 32.34 \\
12 & - & 20.04 \\
13 & - & 14.10 \\
14 & - & - \\
\hline
\end{tabular}

Table 10. Compilation of the ${ }^{13} \mathrm{C}$ NMR data of the halogenated substances from the genus Aplysina. Bromotyrosine Lactone Derivatives.

\begin{tabular}{ccccc}
\hline Position & $\mathbf{2 5}$ & $\mathbf{2 6}$ & $\mathbf{2 7}$ & $\mathbf{2 8}$ \\
\hline 1 & 89.1 & 148.85 & 154.29 & 161.3 \\
2 & 135.5 & 109.12 & 100.74 & 106.7 \\
3 & 119.3 & 103.75 & 161.97 & 155.2 \\
4 & 151.2 & 147.16 & 111.80 & 110.8 \\
5 & 107.6 & 103.75 & 125.75 & 135.2 \\
6 & 77.0 & 113.68 & 122.35 & 117.0 \\
7 & 42.2 & 146.35 & 117.53 & 40.6 \\
8 & 173.7 & 165.91 & 165.23 & 164.0 \\
9 & - & 144.60 & - & - \\
10 & - & 128.12 & 150.75 & - \\
11 & - & 128.12 & 25.21 & - \\
12 & - & 19.79 & 23.69 & - \\
$\mathrm{MeO}$ & 61.5 & - & 61.0 & 60.8 \\
\hline
\end{tabular}


Table 11. Compilation of the ${ }^{13} \mathrm{C}$ NMR data of the halogenated substances from the genus Aplysina. Oxazolides.

\begin{tabular}{ccccc}
\hline Position & $\mathbf{2 9}$ & $\mathbf{3 0}$ & $\mathbf{3 1}$ & $\mathbf{3 2}$ \\
\hline 1 & 153.5 & 151.6 & 142.3 & 140.45 \\
2 & 119.3 & 117.6 & 118.2 & 118.63 \\
3 & 131.9 & 130.7 & 130.8 & 131.45 \\
4 & 140.6 & 138.9 & 140.0 & 152.60 \\
5 & 131.9 & 130.7 & 130.8 & 131.45 \\
6 & 119.3 & 117.6 & 142.3 & 118.63 \\
7 & 76.3 & 74.3 & 54.3 & 75.78 \\
8 & 47.9 & 46.9 & 47.0 & 48.42 \\
9 & 159.8 & 158.5 or 158.2 & 157.8 & 159.05 \\
10 & 75.0 & 73.0 & 53.0 & 72.05 \\
11 & 75.5 & 73.5 & 53.55 & \\
12 & 41.7 & 41.1 & 41.47 & 46.97 \\
13 & 160.2 & 161.35 & 158.4 & 161.35 \\
$\mathrm{MeO}$ & - & - & - & 50.25 \\
\hline
\end{tabular}

Table 12. Compilation of the ${ }^{13} \mathrm{C}$ NMR data of the halogenated substances from the genus Aplysina. Spiroisoxazolines.

\begin{tabular}{|c|c|c|c|c|c|c|c|c|c|}
\hline Position & 33 & 34 & 35 & 36 & 37 & 38 & 39 & 40 & 41 \\
\hline 1 & 75.28 & 75.0 & 75.42 & 75.47 & 75.47 & 75.29 & 75.11 & 75.09 & 75.5 \\
\hline 2 & 122.07 & 122.0 & 122.74 & 114.14 & 114.14 & 122.11 & 122.11 & 122.10 & 114.2 \\
\hline 3 & 148.77 & 148.6 & 149.28 & 149.28 & 149.28 & 148.82 & 148.73 & 148.75 & 149.3 \\
\hline 4 & 113.81 & 113.7 & 114.16 & 122.78 & 122.78 & 113.85 & 113.85 & 113.78 & 122.7 \\
\hline 5 & 132.37 & 132.0 & 132.24 & 132.23 & 132.23 & 132.24 & 132.27 & 132.28 & 133.2 \\
\hline 6 & 91.67 & 91.8 & 92.48 & 92.64 & 92.64 & 90.04 & 91.95 & 91.56 & 92.6 \\
\hline 7 & 40.13 & 39.8 & 40.13 & 40.09 & 40.09 & 39.99 & 39.87 & 40.03 & 40.1 \\
\hline 8 & 155.24 & 154.9 & 155.27 & 155.12 & 155.12 & 154.80 & 154.79 & 155.14 & 155.5 \\
\hline 9 & 160.07 & 160.4 & 161.81 & 161.93 & 161.93 & 160.29 & 160.24 & 160.00 & 161.6 \\
\hline $1^{\prime}$ & 75.28 & 75.1 & 75.42 & 75.47 & 75.47 & 75.29 & 75.18 & 75.20 & 75.5 \\
\hline $2^{\prime}$ & 122.07 & 122.1 & 122.74 & 114.14 & 114.14 & 122.11 & 122.11 & 122.10 & 114.2 \\
\hline $3^{\prime}$ & 148.77 & 148.6 & 149.28 & 149.28 & 149.28 & 148.82 & 148.74 & 148.75 & 149.3 \\
\hline $4^{\prime}$ & 113.81 & 113.7 & 114.16 & 122.78 & 122.78 & 113.85 & 113.85 & 113.78 & 122.7 \\
\hline $5^{\prime}$ & 132.30 & 132.1 & 132.16 & 133.21 & 133.21 & 132.23 & 132.28 & 132.28 & 133.2 \\
\hline $6^{\prime}$ & 91.67 & 91.8 & 92.48 & 92.54 & 92.54 & 91.86 & 91.59 & 91.76 & 92.6 \\
\hline $7^{\prime}$ & 40.09 & 39.9 & 40.04 & 40.03 & 40.03 & 39.89 & 40.09 & 40.08 & 40.1 \\
\hline $8^{\prime}$ & 155.14 & 155.0 & 154.12 & 154.92 & 154.92 & 155.16 & 155.16 & 155.16 & 155.5 \\
\hline $9^{\prime}$ & 160.01 & 160.5 & 161.55 & 161.80 & 161.80 & 160.48 & 160.03 & 160.41 & 161.6 \\
\hline 10 & 37.47 & 43.4 & 37.94 & 49.87 & 49.87 & 47.52 & 47.46 & 43.49 & 38.4 \\
\hline 11 & 30.55 & 69.7 & 30.59 & 207.84 & 207.84 & 200.82 & 200.87 & 69.68 & 26.1 \\
\hline 12 & 72.16 & 75.7 & 71.61 & 75.73 & 75.73 & 76.52 & 76.48 & 75.79 & 26.1 \\
\hline 13 & 152.30 & 152.5 & 153.56 & 43.43 & 43.43 & 151.93 & 151.23 & 151.97 & 38.4 \\
\hline 14 & 118.48 & 118.3 & 118.99 & - & - & 118.11 & 118.07 & 118.31 & - \\
\hline 15 & 134.06 & 131.3 & 131.69 & - & - & 131.55 & 134.21 & 134.14 & - \\
\hline 16 & 139.63 & 142.9 & 143.06 & - & - & 143.93 & 140.53 & 139.88 & - \\
\hline
\end{tabular}


Table 12. Cont.

\begin{tabular}{|c|c|c|c|c|c|c|c|c|c|}
\hline 17 & 134.06 & 131.3 & 131.69 & - & - & 131.55 & 134.21 & 134.14 & - \\
\hline 18 & 118.48 & 118.3 & 118.99 & - & - & 118.11 & 118.07 & 118.31 & - \\
\hline 19 & 34.75 & 71.3 & 72.18 & - & - & 71.46 & 34.74 & 34.71 & - \\
\hline 20 & 40.96 & 47.5 & 47.63 & - & - & 47.60 & 40.85 & 40.91 & - \\
\hline $\mathrm{MeO}$ & 60.21 & 60.2 & 60.35 & 60.39 & 60.39 & 60.23 & 60.19 & 60.19 & 60.4 \\
\hline Position & 42 & 43 & 44 & 45 & 46 & 49 & 50 & 51 & 52 \\
\hline 1 & 72.4 & 75.41 & 75.50 & 74.60 & 74.73 & 73.55 & 73.8 & 74.67 & 73.55 \\
\hline 2 & 119.6 & 114.24 & 120.80 & 121.66 & 121.80 & 120.81 & 121.4 & 121.85 & 120.88 \\
\hline 3 & 145.9 & 149.29 & 149.31 & 147.92 & 148.06 & 147.15 & 147.7 & 148.06 & 147.13 \\
\hline 4 & 111.9 & 122.75 & 114.15 & 115.16 & 115.20 & 113.09 & 113.1 & 115.20 & 113.08 \\
\hline 5 & 130.1 & 132.30 & 131.32 & 132.15 & 132.36 & 131.25 & 130.6 & 132.30 & 131.20 \\
\hline 6 & 89.0 & 92.39 & 92.63 & 91.72 & 91.87 & 90.32 & 91.9 & 91.87 & 90.50 \\
\hline 7 & 38.5 & 40.26 & 40.21 & 40.27 & 40.30 & 42.53 & 38.7 & 40.30 & 39.70 \\
\hline 8 & 153.3 & 155.34 & 155.29 & 155.10 & 155.17 & 154.47 & 153.9 & 155.18 & 154.37 \\
\hline 9 & 157.4 & 161.44 & 161.81 & 160.05 & 160.52 & 158.98 & 160.0 & 160.46 & 159.11 \\
\hline $1^{\prime}$ & 72.4 & 75.51 & 75.50 & 74.67 & 74.73 & 73.55 & 73.8 & 74.67 & 73.55 \\
\hline $2^{\prime}$ & 119.6 & 114.24 & 120.80 & 121.66 & 121.80 & 120.81 & 121.3 & 121.85 & 20.84 \\
\hline $3^{\prime}$ & 145.9 & 149.29 & 149.31 & 147.92 & 148.06 & 147.15 & 147.7 & 148.06 & 147.11 \\
\hline $4^{\prime}$ & 111.9 & 122.75 & 114.15 & 115.16 & 115.20 & 113.09 & 113.1 & 115.20 & 113.08 \\
\hline $5^{\prime}$ & 130.1 & 132.30 & 132.25 & 132.31 & 132.36 & 131.25 & 130.6 & 132.30 & 131.20 \\
\hline $6^{\prime}$ & 88.9 & 92.45 & 92.36 & 91.78 & 91.93 & 90.32 & 91.8 & 91.13 & 90.24 \\
\hline $7^{\prime}$ & 38.5 & 40.26 & 40.04 & 40.27 & 40.30 & 42.53 & 38.7 & 40.16 & 39.45 \\
\hline $8^{\prime}$ & 153.4 & 155.41 & 154.93 & 155.23 & 155.25 & 154.47 & 153.9 & 154.77 & 154.05 \\
\hline $9^{\prime}$ & 157.4 & 161.57 & 161.81 & 160.44 & 160.52 & 158.98 & 160.0 & 160.56 & 158.86 \\
\hline 10 & 44.2 & 39.07 & 49.17 & 37.13 & 43.95 & 39.04 & 36.2 & 47.49 & 48.52 \\
\hline 11 & $\begin{array}{c}67.2 \\
(65.7)^{\mathrm{c}}\end{array}$ & 33.92 & 206.18 & 30.37 & 69.47 & 71.04 & 68.0 & 201.34 & 204.43 \\
\hline 12 & 30.6 & 33.51 & 37.57 & 71.51 & 76.13 & 71.04 & 45.0 & 76.32 & 38.59 \\
\hline 13 & 24.0 & 135.87 & 24.11 & 152.27 & 152.29 & 39.02 & 33.6 & 151.22 & 33.82 \\
\hline 14 & $\begin{array}{c}35.0 \\
(32.5)^{\mathrm{c}}\end{array}$ & 150.68 & 39.61 & 118.35 & 118.42 & - & - & 118.06 & - \\
\hline 15 & - & 111.32 & - & 130.90 & 131.09 & - & - & 131.10 & - \\
\hline 16 & - & 126.67 & - & 143.35 & 143.52 & - & - & 144.23 & - \\
\hline 17 & - & 149.52 & - & 130.90 & 131.09 & - & - & 131.10 & - \\
\hline 18 & - & 126.09 & - & 118.35 & 118.42 & - & - & 118.06 & - \\
\hline 19 & - & 23.09 & - & 70.70 & 69.47 & - & - & 70.70 & - \\
\hline 20 & - & 30.10 & - & 47.99 & 48.15 & - & - & 48. 01 & - \\
\hline 21 & - & 40.03 & - & - & - & - & - & - & - \\
\hline $\mathrm{MeO}$ & 58.4 & 60.44 & 60.38 & 59.75 & 59.86 & 59,63 & 60.0 & 59.86 & 59.63 \\
\hline Position & 53 & 54 & 55 & 56 & 57 & 58 & 59 & 60 & 61 \\
\hline 1 & 74.1 & 75.3 & 75.2 & 75.0 & 74.73 & 74.5 & 74.0 & 74.5 & 68.5 \\
\hline 2 & 113.5 & 113.9 & 113.9 & 57.4 & 57.11 & 57.2 & 55.0 & 57.2 & 54.6 \\
\hline 3 & 147.6 & 148.7 & 148.7 & 183.7 & 183.51 & 184.1 & 184.1 & 184.2 & 183.0 \\
\hline 4 & 121.2 & 122.0 & 122.0 & 122.5 & 122.45 & 122.7 & 123.0 & 122.7 & 123.2 \\
\hline
\end{tabular}


Table 12. Cont.

\begin{tabular}{|c|c|c|c|c|c|c|c|c|c|}
\hline 5 & 131.7 & 132.4 & 132.4 & 149.5 & 149.09 & 149.3 & 146.2 & 149.4 & 146.3 \\
\hline 6 & 90.7 & 91.5 & 91.5 & 91.4 & 91.67 & 91.5 & 90.5 & 91.4 & 90.5 \\
\hline 7 & 40.0 & 40.3 & 40.2 & 38.6 & 38.24 & 38.2 & 41.4 & 38.2 & 41.4 \\
\hline 8 & 155.0 & 155.4 & 155.3 & 154.9 & 154.53 & 154.8 & 155.5 & 154.8 & 155.5 \\
\hline 9 & 159.3 & 159.9 & 159.9 & 159.7 & 159.76 & 160.1 & 160.1 & 160.1 & 159.7 \\
\hline $1^{\prime}$ & 74.1 & 75.1 & 75.0 & 75.0 & 74.78 & 74.5 & 74.0 & 74.5 & 68.5 \\
\hline $2^{\prime}$ & 113.5 & 57.4 & 57.5 & 57.4 & 57.07 & 57.2 & 55.0 & 57.2 & 54.6 \\
\hline $3^{\prime}$ & 147.6 & 183.7 & 183.7 & 183.7 & 183.51 & 184.1 & 184.1 & 184.2 & 183.0 \\
\hline $4^{\prime}$ & 121.2 & 122.5 & 122.5 & 122.5 & 122.36 & 122.7 & 123.0 & 122.7 & 123.2 \\
\hline $5^{\prime}$ & 131.7 & 149.5 & 149.5 & 149.5 & 149.17 & 149.3 & 146.2 & 149.4 & 146.3 \\
\hline $6^{\prime}$ & 90.7 & 91.5 & 91.5 & 91.4 & 91.42 & 91.5 & 90.5 & 91.4 & 90.5 \\
\hline $7^{\prime}$ & 40.0 & 38.6 & 38.6 & 38.6 & 38.10 & 38.2 & 41.4 & 38.2 & 41.4 \\
\hline $8^{\prime}$ & 155.0 & 154.9 & 154.9 & 154.9 & 154.27 & 154.8 & 155.5 & 154.8 & 155.5 \\
\hline $9^{\prime}$ & 159.3 & 159.7 & 159.7 & 159.7 & 159.64 & 160.1 & 160.1 & 160.1 & 159.7 \\
\hline 10 & 45.7 & 39.7 & 39.5 & 39.7 & 49.17 & 45.9 & 45.9 & 45.9 & 45.9 \\
\hline 11 & $\begin{array}{c}67.4 \text { and } \\
68.9\end{array}$ & 29.7 & 27.5 & 29.7 & 204.70 & 68.8 & 68.8 & 70.3 & 70.3 \\
\hline 12 & 32.2 & 24.7 & 27.5 & 24.7 & 39.63 & 34.6 & 34.6 & 32.4 & 32.4 \\
\hline 13 & 25.5 & 29.7 & 39.5 & 29.7 & 34.78 & 37.0 & 37.0 & 26.0 & 26.0 \\
\hline 14 & 36.6 & 39.7 & - & 39.7 & - & - & - & 37.0 & 37.0 \\
\hline 15 & 34.4 & - & - & - & - & - & - & - & - \\
\hline 16 & 39.4 & - & - & - & - & - & - & - & - \\
\hline $\mathrm{MeO}$ & 60.1 & 60.2 & 60.2 & - & - & - & - & - & - \\
\hline Position & 62 & 63 & 64 & 65 & 66 & 67 & 68 & 69 & 70 \\
\hline 1 & 56.9 & 75.3 & 73.55 & 73.56 & 73.57 & 75.45 & 73.44 & 75.40 & 73.6 \\
\hline 2 & 53.0 & 113.8 & 120.80 & 120.79 & 120.86 & 122.68 & 120.64 & 122.72 & 120.8 \\
\hline 3 & 186.0 & 149.0 & 147.13 & 147.12 & 147.16 & 149.23 & 147.07 & 149.23 & 147.3 \\
\hline 4 & 122.8 & 122.0 & 113.04 & 113.04 & 113.11 & 114.12 & 113.00 & 114.10 & 113.1 \\
\hline 5 & 143.7 & 132.4 & 131.19 & 131.21 & 131.23 & 132.21 & 131.22 & 132.14 & 131.2 \\
\hline 6 & 84.0 & 91.5 & 90.16 & 90.24 & 90.52 & 92.39 & 90.12 & 92.55 & 90.2 \\
\hline 7 & 43.6 & 40.2 & 39.50 & 39.50 & 39.33 & 40.19 & 39.50 & 40.06 & 39.3 \\
\hline 8 & 154.9 & 155.3 & 154.34 & 154.34 & 154.08 & 155.32 & 154.44 & 155.11 & 154.3 \\
\hline 9 & 158.3 & 159.3 & 158.37 & 158.87 & 159.13 & 161.33 & 158.82 & 161.61 & 158.9 \\
\hline $1^{\prime}$ & 56.9 & 58.1 & - & - & - & - & - & - & - \\
\hline $2^{\prime}$ & 53.0 & 54.2 & - & - & - & - & - & - & - \\
\hline $3^{\prime}$ & 186.0 & 186.0 & - & - & - & - & - & - & - \\
\hline $4^{\prime}$ & 122.8 & 124.1 & - & - & - & - & - & - & - \\
\hline $5^{\prime}$ & 143.7 & 144.1 & - & - & - & - & - & - & - \\
\hline $6^{\prime}$ & 84.0 & 85.3 & - & - & - & - & - & - & - \\
\hline $7^{\prime}$ & 43.6 & 44.5 & - & - & - & - & - & - & - \\
\hline $8^{\prime}$ & 154.9 & 155.6 & - & - & - & - & - & - & - \\
\hline $9^{\prime}$ & 158.3 & 159.9 & - & - & - & - & - & - & - \\
\hline
\end{tabular}


Table 12. Cont.

\begin{tabular}{|c|c|c|c|c|c|c|c|c|c|}
\hline 10 & 38.6 & 39.8 & 33.83 & 49.89 & 48.58 & 39.49 & 38.53 & 37.86 & 36.2 \\
\hline 11 & 26.4 & 29.7 & 38.29 & 205.66 & 205.75 & 123.47 & 28.23 & 30.76 & 29.4 \\
\hline 12 & 26.4 & 24.7 & 205.66 & 38.29 & 39.75 & 122.33 & 23.28 & 72.30 & 71.2 \\
\hline 13 & 38.6 & 29.7 & 49.89 & 33.83 & 35.21 & 130.81 & 27.99 & 153.61 & 150.8 \\
\hline 14 & - & 39.7 & 156.98 & 156.98 & 157.03 & 151.45 & 40.65 & 118.94 & 117.0 \\
\hline 15 & - & - & - & - & - & 118.11 & 157.20 & 131.66 & 133.0 \\
\hline 16 & - & - & - & - & - & - & - & 142.82 & 140.6 \\
\hline 17 & - & - & - & - & - & - & - & 131.66 & 133.0 \\
\hline 18 & - & - & - & - & - & - & - & 118.94 & 117.0 \\
\hline 19 & - & - & - & - & - & - & - & 71.64 & 33.7 \\
\hline 20 & - & - & - & - & - & - & - & 47.62 & 44.7 \\
\hline 21 & - & - & - & - & - & - & - & 173.29 & - \\
\hline 22 & - & - & - & - & - & - & - & 47.62 & - \\
\hline 23 & - & - & - & - & - & - & - & 22.68 & - \\
\hline $\mathrm{MeO}-\mathrm{C}_{3}$ & - & 60.2 & 59.60 & 59.60 & 59.79 & 60.42 & 59.56 & 60.42 & 59.6 \\
\hline $\mathrm{MeO}-\mathrm{C}_{14}$ & - & - & 51.54 & 51.54 & 51.28 & - & - & - & - \\
\hline Position & 71 & & 74 & 75 & 76 & & 79 & 80 & 81 \\
\hline 1 & 75.21 & & 73.4 & 75.5 & 73.57 & & 76.4 & 76.3 & 75.1 \\
\hline 2 & 122.15 & & 120.8 & 114.1 & 120.87 & & 115.1 & 114.9 & 113.8 \\
\hline 3 & 148.78 & & 147.0 & 149.3 & 147.18 & & 150.2 & 150.1 & 149.0 \\
\hline 4 & 113.90 & & 113.3 & 122.8 & 113.08 & & 123.7 & 123.5 & 122.3 \\
\hline 5 & 132.27 & & 131.2 & 132.3 & 131.21 & & 133.2 & 133.0 & 132.3 \\
\hline 6 & 91.84 & & 90.1 & 92.3 & 90.33 & & 93.4 & 93.2 & 92.6 \\
\hline 7 & 40.08 & & 39.7 & 40.2 & 39.26 & & 41.0 & 40.1 & 40.0 \\
\hline 8 & 155.14 & & 154.8 & 155.3 & 154.37 & & 156.0 & 159.5 & 153.3 \\
\hline 9 & 160.42 & & 158.9 & 161.6 & 159.10 & & 162.8 & 162.5 & 161.0 \\
\hline 10 & 47.71 & & 39.4 & 39.9 & 37.66 & & 44.7 & 40.5 & 63.0 \\
\hline 11 & 71.43 & & 24.5 & 29.8 & 24.12 & & 78.4 & - & 14.4 \\
\hline 12 & 138.5 & & 34.5 & 25.6 & 130.79 & & 25.7 & - & - \\
\hline 13 & 111.38 & & 174.7 & 61.9 & 133.84 & & 40.2 & - & - \\
\hline 14 & 130.90 & & - & 190.6 & 116.21 & & 157.5 & - & - \\
\hline 15 & 150.72 & & - & 171.4 & - & & - & - & - \\
\hline 16 & 111.38 & & - & - & - & & - & - & - \\
\hline 17 & 130.90 & & - & - & - & & - & - & - \\
\hline $\mathrm{MeO}-\mathrm{C}_{3}$ & 60.22 & & 59.6 & 60.4 & 59.63 & & 61.3 & 59.2 & 60.8 \\
\hline
\end{tabular}

Although the isoxazoline alkaloids are the group with more ${ }^{13} \mathrm{C}$ NMR data, some chiral centers of this group continue with an undefined stereochemistry due to the incompatibility of using X-ray crystallography techniques, possessing sometimes non-crystalline characteristic [98]. Some positions with ${ }^{13} \mathrm{C}$ NMR data had to be revised because there were mistakes in the numbering of the carbon skeleton in the attribution of values of some positions in this group of alkaloids. 
Table 13. Compilation of the ${ }^{13} \mathrm{C}$ NMR data of the halogenated substances from the genus Aplysina. Verongiabenzenoids.

\begin{tabular}{ccc}
\hline Position & $\mathbf{8 2}$ & $\mathbf{8 3}$ \\
\hline 1 & 154.87 & 152.19 \\
2 & 111.18 & 117.30 \\
3 & 134.63 & 133.35 \\
4 & 129.20 & 135.92 \\
5 & 130.33 & 133.35 \\
6 & 117.68 & 117.30 \\
7 & 29.07 & 26.88 \\
8 & 68.51 & 65.07 \\
$\mathrm{OMe}$ & 59.88 & 60.31 \\
$(\mathrm{Me})_{3} \mathrm{~N}^{+}$ & 53.77 & 52.23 \\
\hline
\end{tabular}

Table 14. Compilation of the ${ }^{13} \mathrm{C}$ NMR data of the halogenated substances from the genus Aplysina. Verongiaquinol.

\begin{tabular}{cccc}
\hline Position & $\mathbf{9 0}$ & $\mathbf{9 7}$ & $\mathbf{9 8}$ \\
\hline 1 & 172.6 & 183.0 & 183.0 \\
2 & 119.9 & 122.7 & 123.7 \\
3 & 153.2 & 151.7 & 146.6 \\
4 & 70.8 & 75.5 & 74.2 \\
5 & 148.8 & 78.4 & 78.9 \\
6 & 127.6 & 56.1 & 57.1 \\
$1^{\prime}$ & - & 116.9 & 116.9 \\
2 & - & 28.4 & 28.4 \\
$\mathrm{CH}_{2}$ & 45.1 & - & - \\
$\mathrm{CONH}_{2}$ & 169.4 & - & - \\
\hline
\end{tabular}

Table 15. Compilation of the ${ }^{13} \mathrm{C}$ NMR data of the halogenated substances from the genus Aplysina. Dibromocyclohexadiene.

\begin{tabular}{cc}
\hline Position & $\mathbf{1 0 1}$ \\
\hline 1 & 86.3 \\
2 & 109.1 \\
3 & 149.7 \\
4 & 113.6 \\
5 & 140.4 \\
6 & 76.5 \\
7 & 42.1 \\
8 & 172.8 \\
9 & 60.2 \\
10 & 60.3 \\
\hline
\end{tabular}

\section{Discussion}

The genus Aplysina belongs to the order Verongida, sponges with a wide variety of metabolites. Sterols [110,111], carotenoids [112], amino acids [113] and rare fatty acids [114] have all been 
isolated from this order. However, the peculiarity of this order is from the ecological and medicinal points of view, in that great production of halogenated substances originates from the metabolism of amino acids such as phenylalanine and tyrosine.

The halogenated substances found in the marine sponges of the genus Aplysina can be classified as: (A) Bromotyramines, (B) Cavernicolins, (C) Hydroverongiaquinols, (D) Bromotyrosineketals, (E) Bromotyrosine lactone derivatives, (F) Oxazolidones, (G) Spiroisoxazolines, (H) Verongiabenzenoids, (I) Verongiaquinols and (J) Dibromociclohexadiens.

\subsection{Chemotaxonomy Importance of Aplysina Sponges}

Although in the past, it was suspected that bromotyrosine compounds were not present in Brazilian Aplysina species [69], nowadays numerous studies have shown the presence of these chemical biomarkers, not only in Brazilian species, but in almost all the Verongida order.

In order to classify the large number of halogenated compounds reviewed in Table 2, for each sponge species, we listed the halogenated compounds under the correlated species. Considering the taxonomic species diagnosis of morphologic variation of spongin fibers is difficult [33], chemical composition can be used as a tool for a more accurate identification. The distribution of the halogenated compounds is widespread in Aplysina genre, and studies show that mainly bromoisoxazoline alkaloids have been found in almost all species. This family of metabolites was usefully employed as a chemical marker for the distinction of some taxa as Aplysina aerophoba and Aplysina cavernicola, two very physically similar species [63], but biochemically different. In another situation, majority of aerothionine was key to identify two subspecies of $A$. fistularis, which split into A. fulva and A. insularis [115].

The similarity between agelorins A and B, isolated from Agelas oroides and produced by Aplysina caissara, was essential to show the two genera, Aplysina and Agelas, have a phylogenetic relationship [76] and 11-epi-fistularin-3 was yielded by Aplysina fulva [98].

The presence of stereo metabolites isolated from Aplysina sponges as derivatives of fistularin-3 discussed by Rogers et al., 2005 [98], provides evidence that enzymatic pathways are non-stereoselective in these sponges.

As can be seen, each kind of genus adds a different profile of metabolites. However, even with a different chemical profile, Aplysina sp. has compounds that give a clue to their evolutionary origin.

\subsection{Bromotyramines}

The substances aplyzanzine A, aplysamine-1 and aplysamine- 2 present a dibromotyramine structural portion, and probably originated in accordance with Evan et al. [82], by amidation with other bromotyrosinated radicals. Moloka'inamine [116] and purealidin C isolated from Psammaplysilla purea [90] are examples of metabolites isolated from sponges of the order Verongida, having dibromotyramine in their structures. According to Carney, free phenolic groups are important precursors nitrile phenolic [96], hence the similarity between methoxylated compound, aplysamine-2, and hydroxylated analogue, psammaplin-A [84], observed by Xynas and Capon, 1989, shows that psammaplin-A may be important precursor aplysimines as much of the fistularin and its derivatives 


\subsection{Cavernicolins}

Figure 1. Metabolism of the bromotyrosine derived metabolites $(\rightarrow$ For the steps clarified in previous studies and $\cdots$ - For the biogenesis hypothesis).

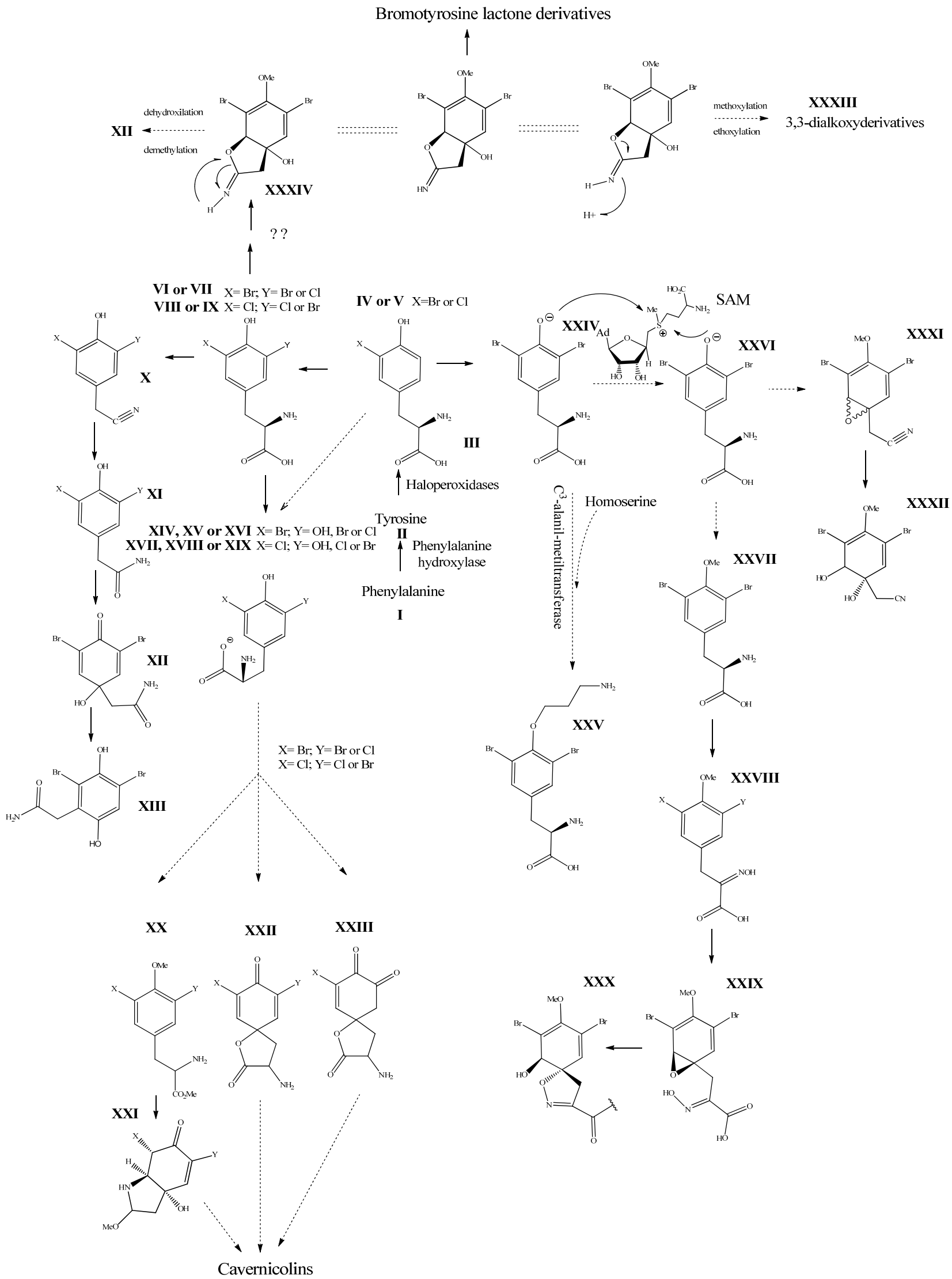


Cavernicolines are $\gamma$ and $\delta$-lactames formed by a residual halogenated tyrosine precursor [81] and also having a bi-cyclic system. The junction of the rings occurs in carbons C-3 and C-4 in ortho position, while in the 7-bromocavernicolenone and in the 7-chlorocavernicolenone, this junction occurs in carbons C-2 and C-4. They can be defined as haloperoxidases with the role of converting either the 3-chloro (V) ortho 3-bromotyrosine (IV) in residues of 3,5-dichloro (VIII) or 3,5-dibromotyrosine (VI) or 3-chloro-5-bromotyrosine (VII) or 3-bromo-5-chlorotyrosine (IX), respectively [80]. These substances have a chiral center at C-2 and their $R$ and $S$ enantiomers are obtained in racemic mixtures, or relatively pure from the genus Aplysina. In the formation of cavernicolines, as to the substitution pattern (ortho or para) it is suggested that the biosynthesis pathway has either a halo-tyrosine (XV, XVI, XVIII and XVIX) or a halo-dopa (XIV and XVVI) intermediary which will form a spirolactone precursor (XXII and XXIII), allowing the formation of intermediaries in racemic or quasi-racemic mixtures. The absence of control in the absolute stereochemistry of this class is intrinsic to phenol oxidative coupling [81,117]. It is noteworthy that experimental observations [118] show that 3,5-dibromo-4-methoxyphenylalanine methyl ester (XX) in reaction in an anodic oxidative medium form a more appropriate intermediary (XXI) than the spirolactone, originating derivatives similar to the stereoisomers of the cavernicolines with considerable yields (See Figure 1).

\subsection{Hydroverongiaquinols}

The hydroverongiaquinols are 2,6-bromotyrosine phenolic derivatives. Both the hydroverongiaquinols and the verongiabenzenoids are important mediators in biosynthesis of other classes of bromotyrosine metabolites. The verongiabenzenoids are part of the biosynthesis of isoxazoline alkaloids, and hydroverongiaquinols are important precursors in the formation of metabolites which need free phenolic groups to convert themselves into $\alpha$-oximine substances, such as the phenolic nitriles. However, phenolic nitriles have not been found in the genus Aplysina, they are found in the genus Ianthella where substances like the bastadins, are important chemotaxonomic markers for the genus [119].

\subsection{Bromotyrosineketals}

The bromotyrosineketals have a 3,5-dibromocyclohexa-2,5-dienyl ketal skeleton system. Literature shows that the dimethoxy and methoxy-ethoxy ketals (XXXII) isolated from Aplysina fistularis and from Aplysina cauliformis $[92,93,120]$, are artifacts formed by the oxidation of dienone, since the dimethoxy form is obtained as a mixture of diastereoisomers [94,95], both showing antibacterial activity. Further evidence that they are artifacts is the formation of dimethoxy and methoxy-ethoxy ketals which can be explained as being formed from an arene (dienone intermediary XII), which suffers 1,4 additions of methanol, water or ethanol (Figure 2), and displayed a reaction described by Kasperek et al. $[94,95,121]$. However, the methoxy-butoxy and methoxy-pentoxy ketals isolated from Aplysina thiona [109] are not considered reaction products. Aplysinketal A was isolated only in the form of diastereoisomers, and the absence of the dimethoxy ketal indicates the non-existence of reactions during the extraction process [109]. It has been suggested that the formation of the $\mathrm{C}_{4}$ and $\mathrm{C}_{5}$ chains are formed via lysine and ornithine respectively (Figure 2) [104,114]. 
Figure 2. Spiroisoxazoline and acetal formation as production artefacts.

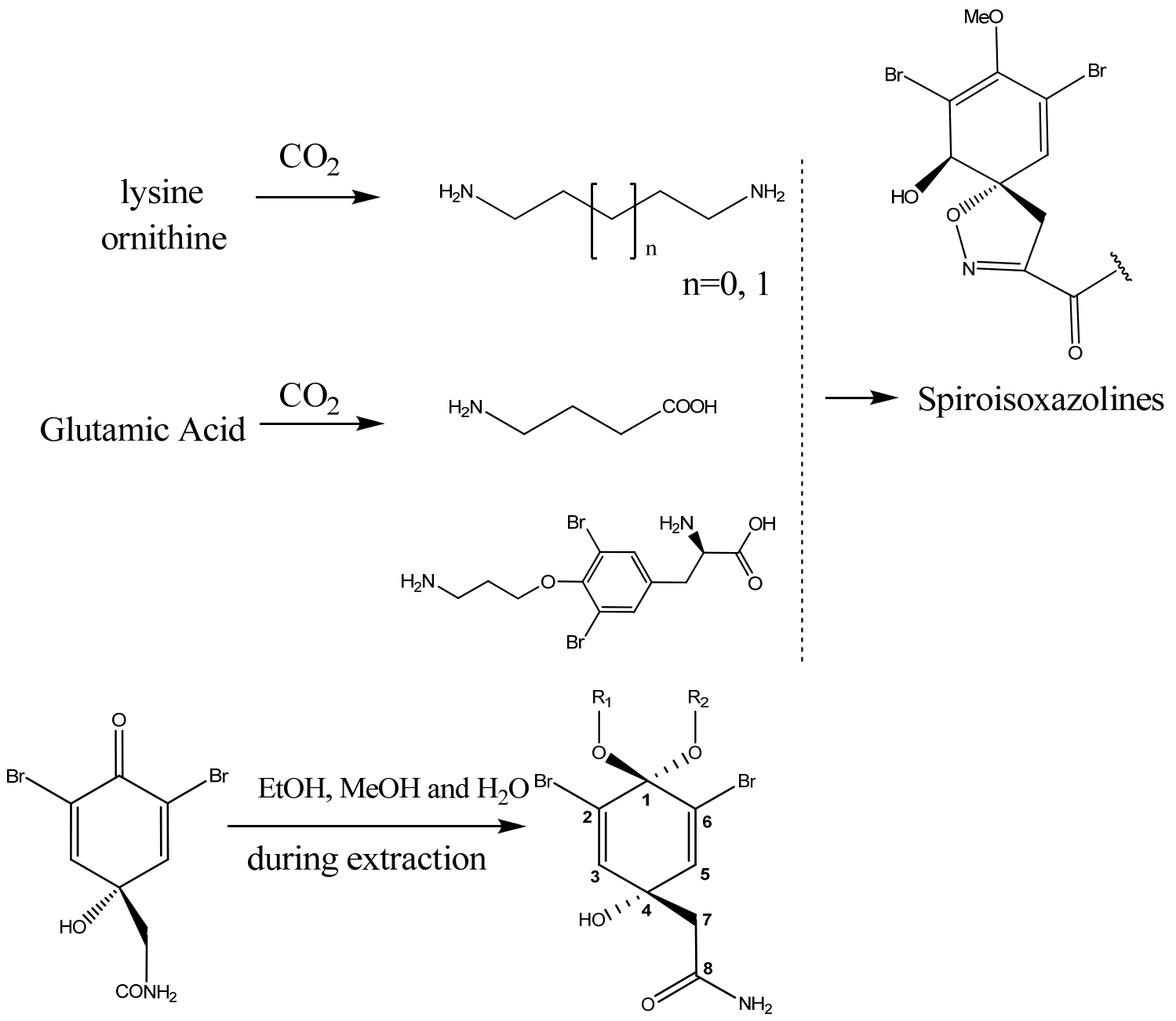

3,3-dialcoholderivatives

\subsection{Bromotyrosine Lactone Derivatives}

The bromotyrosine lactone derivatives, with the exception of aplysinimine which is an imine, are five member lactones condensated with 3,5-dibromotyrosine residues (XXIV). Aeroplysinin-2 is different from the others because it has a cyclodiene group instead of an aromatic ring, while aplysinadiene presents a cis-trans diene side chain. It is proposed that the biosynthesis of this class of substances has an imine-ether (XXXIV) as initial intermediary, a hydroxylated derivative of aplysinimine. This derivative will either suffer a tertiary alcohol dehydration to form aplysinimine [109,120] or will follow other biosynthetic pathways similarly to bromotyrosine lactones as aeroplysinin-2, or the verongiabenzenoids and verongiaquinols, in this case the intermediary suffers dehydroxylation and demethylation and can form artifacts such as dimethoxys and methoxy-ethoxy ketals. [122]. Aplysinolide is considered an artifact, since it possesses an $\alpha, \beta$-unsaturated side chain which is uncommon to find linked to a lactone ring. In accordance to Cruz et al. [109] this substance can be formed by combining aplysinimine with $\mathrm{Me}_{2} \mathrm{CO}$ during the purification process (see Figure 1). 


\subsection{Oxazolidones}

Oxazolidones are very common in Aplysina, but just two types are found: diastereoisomers of bisoxazolidone and methoxy derivative. This derivative presents two chiral centers with different stereochemistries for different species. Studies show that these bisoxazolidone's isomers have a relative configuration of $7 S^{*}, 11 R^{*}$ [97] and $11 R^{*}, 7 S^{*}$ [106], as determined by comparison with bisoxazolidone isolated from Ascidia Clavelina oblonga [123] and an absolute configuration $R, R$ [64] obtained by X-ray crystallography. There are evidences that fistularin-3 degradation promotes bisoxazolidone and aeroplysinin-1 production [13].

\subsection{Spiroisoxazolines}

The spiroisoxazolines also known as isoxazoline alkaloids form the biggest group of Aplysina and Verongid order metabolites. They are divided into two structure types: mono-spirocyclohexadienylisoxazolines and bis-spirocyclohexadienylisoxazolines [124].

The chemical structure of mono-spirocyclohexadienylisoxazolines (nuclei G7, G8) have essentially one spirocyclohexadienylisoxazoline ring bonded to a 1-6 carbon side chain with exception of the spiroisoxazoline acid ester (nucleus G9) [83] considered an artifact of ethanolic condensation from spiroisoxazoline acid [125]. The bis-spirocyclohexadienylisoxazolines (nuclei G1, G2) can present the same ring, which in the more common example is bonded to a 3-11 carbon side chain.

This side chain is bonded to another spirocyclohexadienylisoxazoline ring [124]. In other spiroisoxazolines, the rings suffer oxidation of the methoxy group forming a cyclohexenone (nuclei G3, G4) [74,76,101]. Cyclohexenone also suffers hydroxyl or bromine oxidations originating cyclohexenone epoxide between carbons 1 and 2 and forming oxaspirocyclohexenonylisoxazolines (nuclei G5, G6) [100]

The biosynthetic pathway for spiroisoxazolines needs tyrosine intermediates with $O$-methyl groups (XXVII), which are metabolized to form oxime grouped intermediates (XXVIII) and shortly thereafter form other intermediaries with arene oxide (XXIX). The nucleophilic attack of the oxime over either the epoxide or the phenol originates by breaking the epoxide which forms the isoxazole ring (XXX) [96]. The $\mathrm{C}_{4}$ or $\mathrm{C}_{5}$ side chains that extend out of the ring such as in aerothionin and inhomoaerothionin are produced via ornithine and lysine respectively (Figure 2) $[70,94,95,126]$. However, when the side chains present a 4-aminobutanoic substituent it is suspected that the amino acid involved is glutamic acid (Figure 2) [99]. In some spiroisoxazolines such as fistularin-3, and araplysillin $\mathrm{N}^{9}$-sulfamate, there is a 3-amino-1-propanol connector which binds itself to other structures and probably has as a precursor of its biosynthesis a decarboxylated product of the uncommon amino acid, homoserine. This amino acid is an intermediary for the enzyme S-adenosylmethionine (SAM). It is suggested that the SAM is involved in $\mathrm{S}_{\mathrm{N}} 2$ substitutions of hydroxyl of 3,5-dibromotyrosine (DBT) (XXIV), making it susceptible to the formation of methoxyl groups by methyltransferase and $O$-alkylated bonds via other enzymes. The enzyme responsible for $O$-alkylations is putative $\mathrm{C}^{3}$-alanil-methyltransferase which allows 3-amino-1-propanol connector bonding to the DBT residue, forming spiroisoxazoline residue complexes with large molecular masses (XXV) which are then incorporated in the isoxazoline rings [99]. The alkaloid archerine, a dimer of two imidazole rings, is probably formed by oxidative coupling [1 +1$]$ of two aerophobin-2 molecules [73]. 
In sponges of the order Verongida, the spherule cells have the capacity of stocking and secreting isoxazoline alkaloids, which are modified by enzymes located in distinct locations $[127,128]$. The extracts of the majority of the sponges belonging to the genus Aplysina, when tested, show that their enzymes convert brominated isoxazoline alkaloids into aeroplysinin-1 and dienones. Sponges of other orders are unable to perform this biotransformation [129]. Puyana et al. [130] demonstrated that there is no aeroplysinin-1 and dienones production when there is a decrease in the amount of spiroisoxazolines. The ecological function of this enzymatic activation is microbial pathogen growth inhibition and the repellent odor, which decreases the predatory search by fishes [129].

Although the agelocaissarines A1, A2, B1 and B2 were initially considered production artifacts as pairs of stereoisomers, this was later modified by the observation of in vitro experiments showing the absence of substances with relative stereochemistries different to those found in the work [76].

In therapeutics these substances demonstrate tumor cell cytotoxic [22], antimicrobial [15] and antihistamine [73] activity.

Spiroisoxazolines vary in different species, but also inside the single species. While A. fulva produces aerothionin as its major component (0.11\%) [34], this same substance is not present in A. insularis [74] and in A. fulva it appears with a larger amount (0.52\%) [102]. Nuñez et al. [97], affirms that this chemical variation may be due to either different extraction and isolation techniques, or to biological diversity of the areas in which the sponges are collected. This chemical distinction led to reinforce the hypothesis that Aplysina aerophoba and Aplysina carvernicola have metabolic differences and that $A$. aerophoba, erroneously identified in the work of Cimino et al. [67], was in fact Aplysina cavernicola.

The presence of hemi-fistularin, isolated together with 11-oxofistularin-3, begs us to question whether the first is a precursor of 11-oxofistularin-3 biogenesis, or a degradation product [72]. Fistularin-3 shows another type of variability. Besides having stereoisomers of $(+)$ fistularin- 3 , such as $(+)$-isofistularin-3 and 11-epi-fistularin-3, the chemical composition of sponges contains them in irregular proportions, which makes it difficult to determine through optical rotation. In order to determine the absolute configuration of fistularin-3 and its stereoisomers, a microscale analysis with Marfey's reagent, has been used that led to the formation of stable reaction products analyzed by LC-MS [98].

\subsection{Verongiabenzenoids}

The verongiabenzenoids are aromatic methoxylated substances which present a skeleton with a 2,6-dibromomethoxybenzene nucleus. Biogenetically methoxylated, some verongiabenzenoids can form isoxazolines [13] or epoxide intermediaries from the arene oxide, which leads to forming other verongiabenzenoids [96].

\subsection{Verongiaquinols}

The chemical structure of the verongiaquinols is either a cyclohexadien-2-one or cyclohexadien-2,6-one system, with either bromine or chlorine substituents in positions 2 and 6 and hydroxyls on carbons C-3 and C-4. They also may suffer ramifications on carbon C-4. The verongiaquinols seem to be related to degradation steps of tyrosine metabolism, as is the case of 
dienone (XII). The degradation of bromotyrosine substances such as iso-fistularin-1 and aerophobin-2 after mechanical injury led to the formation of aeroplysinin-1 (XXXII) and (XII). It is also noteworthy that the extraction of frozen sponges and consequent exposure to alkaline sea water will form dienones (XII) $[97,130,131]$. Besides being integrated at the metabolic level, dienone and aeroplysinin-1 have an important defense role for sponges: cytotoxic, algicidic, molluscicide and antibacterial activity have been reported [131,132].

Other verongiaquinols such as 2,6-dibromoquinone have been reported to inhibit the enzyme RNA polymerase II, blocking the initiation of the chain, but not its elongation [133].

It is not known for sure if $\left(1^{\prime} R, 5^{\prime} S, 6^{\prime} S\right)$ and $\left(1^{\prime} R, 5^{\prime} R, 6^{\prime} S\right)-2-\left(3^{\prime}-5^{\prime}\right.$-dibromo-1'-6'-dihydroxy-4'-oxocyclohex-2'-enyl)-acetonitrile are simple artifacts, as aeroplysinin-1 is able to form them in the presence of acetone by keto-enol tautomerism. However, these acetonitriles are not normally produced as metabolites of aeroplysinin-1 (XXXII) in other species [27].

\subsection{Dibromocyclohexadienes}

This group is comprised of two substances which present a 1,2-dihydroarene-1,2-diol and may have their biogenesis via an arene oxide (XXXI) in agreement with their stereochemistry [94,95]. Aeroplysinin-1 (XXXII) is a nitrilated substance found in dextro and levorotatory forms. The dextrorotatory isomer (+) aeroplysinin-1, has been obtained from Aplysina aerophoba [70], Aiolocroia crassa, Verongula rigida, Aplysina archeri [71] and Psammoposilla purpurea [134]. (-) Aeroplysinin-1 has been found in Ianthella ardis [135] and Verongula gigantean [71]. The metabolic degradation of bis-oxazolidone, isofistularin-3, aplysinamisin-1 and aerophobin-2 is known to be an important source of aeroplysinin-1 [13]. In terms of pharmacological activity, aeroplysinin-1 is a versatile substance which has demonstrated cytotoxic [134], antiprotozoal [136] and antiangiogenic [137] activities. Aplysifulvin is one of the most recently isolated substances from the sponge A. fulva [97]. It possesses only two methoxies and no ethoxies, and since no ethoxy derivatives were detected, the possibility that aplysifulvin is an artifact has been discarded. Hypothetically, the chemical structure of aplysifulvin suggests that the 3,3-dialkoxy ketals (with OMe and OEt groups) previously described are artifacts [97].

\subsection{Structural Elucidation}

This section describes the compilation of the ${ }^{13} \mathrm{C}$ chemical shifts of halogenated compounds of the genus Aplysina. All compounds compiled in this review-bromotyramines (1-4), cavernicolins (5-17), hydroverongiaquinols (18-20), bromotyrosineketals (21-24), bromotyrosine lactone derivatives (25-28), oxazolidones (29-32), spiroisoxazolines (33-81), verongiabenzenoids (82-86), verongiaquinols (87-99) and dibromocyclohexadienes (100-101) - have in common 3,5-halotyrosine or halophenylalanine derivatives.

Research data shows that works from the decades of 1970, 1980 and 1990 show little or no information of ${ }^{13} \mathrm{C}$ NMR as compounds (95-97) whose structural elucidation was done by mass spectrometry (MS) and ${ }^{1} \mathrm{H}$ NMR spectroscopy analysis and reactions of structural identification.

The bromotyramine family skeleton can be recognized by the typical ${ }^{1} \mathrm{H}-\mathrm{NMR}$ signals, as for example, in the case of compound 1: a singlet for aromatic protons H-2 and H-6 ( $\delta$ 7.62) and four 
triples $(\delta 3.02,2 \mathrm{H}, 8.0 \mathrm{~Hz} ; \delta 3.50,2 \mathrm{H}, 8.0 \mathrm{~Hz} ; \delta 4.12,2 \mathrm{H}, 5.5 \mathrm{~Hz} ; \delta 3.22,2 \mathrm{H}, 5.5 \mathrm{~Hz})$ attributed to H-7, H-8, H-1', H-3' respectively [84]. When NH-3' has low electron density as compound 1 positively charged and compound 2 close to electrophilic oxime group, the ${ }^{13} \mathrm{C}-\mathrm{NMR}$ signals shift to downfield compared to compounds $\mathbf{3}$ and $\mathbf{4}$, whose substituents are methyl groups [84].

Typical ${ }^{1} \mathrm{H}$ NMR data from the cavernicolin class are, in the case of compound $\mathbf{5}$, for H-3 $\delta 4.05$ (dd, $\mathrm{J}=10.1 \mathrm{~Hz}$ and $\mathrm{J}=1.5 \mathrm{~Hz}$ ), a singlet for $\mathrm{H}-5$ at $\delta 7.3$ and the signals for $\mathrm{NH}$ and $\mathrm{OH}$ at 6.9 (s) and 4.4 (s) respectively [83]. ${ }^{13} \mathrm{C}$ NMR data shows that chlorinated carbon have their signals at downfield shifts in relation to bromine carbons as it can be seen, for example, comparing compounds 5 and 6 with 9 and compound $\mathbf{1 1}$ with $\mathbf{1 2}$.

Biosynthetically, verongiaquinol metabolites are the oxidized form of hydroverongiaquinols, considered as a hydroquinone precursor [124,138]. Therefore, the basic difference in the ${ }^{13} \mathrm{C} N M R$ between these two classes is the downfield signal of ketone carbon C-1 ( $\delta$ 172.6) of compound 91 compared with hydroxylated C-1 ( $\delta$ 150.0) of compound 20 [97].

Data analysis of the ${ }^{13} \mathrm{C}$ NMR bromotyrosine lactone family brominated aromatic show carbon signals have more shielded signals compared to the other aromatic signals of the ring. The more is the unsaturated side branching at C-7, more deshielded are the signals of the lactone ring, with the increasing order of introducing the compounds 28, 27 and 26 [64,109].

The carbons of the spiroisoxazolinic system of most compounds with cores $G_{1}, G_{2}, G_{7}, G_{8}$ and $G_{9}$ acquire values which become a standard set of values, with the exception of carbons $\mathrm{C}-2$ and $\mathrm{C} 4$ values, which seem to be mistakenly exchanged one for another at $\delta 120$ or $\delta 114$, being the correct value of $\delta 120$ for carbon C-2 due to the proximity of the hydroxyl and the C-4 for $\delta 114$.

The mono and bis-spiroisoxazolinic ring systems could be distinguished by double ${ }^{1} \mathrm{H}-\mathrm{NMR}$ shield and deshield signals for the two rings, for example, compound 50 shows signals at $\delta 4.16(1 \mathrm{H}$, d, $\mathrm{J}=8.3 \mathrm{~Hz})$ for $\mathrm{H}-1$ and $\delta 4.58(1 \mathrm{H}, \mathrm{d}, \mathrm{J}=7.9 \mathrm{~Hz})$ for $\mathrm{H} 1^{\prime}$. A typical methylene signal at $\delta 4.43 ; 3.47$ $(2 \mathrm{H}, \mathrm{ABq}, \mathrm{J}=18.2)$ is attributed to the isoxazol ring protons $\mathrm{H}-7,7$ ' for this compound [29]. Today the most used techniques to elucidate absolute stereochemistry of the rings are ${ }^{1} \mathrm{H}-\mathrm{NMR}$ spectrum analysis and molecular modeling using both MM2 and MOPAC protocols of the Chem3D software [76], and also NOE-difference spectroscopy studies [100].

${ }^{13} \mathrm{C}$ data reveals spiroisoxazoline ring systems have distinguished shifts. The difference between cyclohexadienone $\left(\mathrm{G}_{1}, \mathrm{G}_{2}, \mathrm{G}_{7}, \mathrm{G}_{8}\right.$ and $\left.\mathrm{G}_{9}\right)$ and cyclohexenone $\left(\mathrm{G}_{3}\right.$ and $\left.\mathrm{G}_{4}\right)$ systems are two chemical shifts at downfield for C-3' and C-5' (54-55) and one at upfield for C-2' in $\mathrm{G}_{3}$ (56-61), and the same shifts for $\mathrm{C}-3,-33^{\prime}, \mathrm{C}-5,-5^{\prime}, \mathrm{C}-2,-2^{\prime}$ in $\mathrm{G}_{4}(\mathbf{5 6}-\mathbf{6 1})$. The epoxide group in $\mathrm{G}_{5}$ and $\mathrm{G}_{6}$ can be characterized by the same shifts plus two differences: a strong shift at downfield for C-3 and C-3' in $\mathrm{G}_{5}$ and $\mathrm{C}-3^{\prime}$ in $\mathrm{G}_{6}$. The other difference involves three chemical shifts at upfield for C-1, 1', C-2, 2' and C-6, 6' of 62 and for C-1', C-2' and C-6' of 63. Some ${ }^{13} \mathrm{C}$ data as $\delta 67.2$ and $\delta 65.7$ attributed to chiral C-11 of compound $\mathbf{4 2}$ still remain with its stereochemistry unsolved [76].

According to Kossuga, 2004 [123], to determine the configuration of the relative stereochemistry of bis-oxazolidone uses $[\alpha]$ of $(7 R, 11 R)$ bis-oxazolidone with absolute stereochemistry [64]. It was possible to determine the relative configuration of bis-oxazolidones $\left(7^{*} R, 11^{*} S\right)$ and $\left(11^{*} R, 7^{*} S\right)$ isolated in previous works as can be seen in Table $2[63,65,109]$. 


\section{Conclusions}

The genus Aplysina is one of the richest in secondary metabolites, which have been cataloged in 14 species from the Aplysinidae family. Most classes of compounds mentioned here present themselves brominated, and, despite the large number of species of the genus Aplysina, many have not been studied chemically. The halogenated compounds found in marine sponges of this genus were classified into: (A) Bromotyramines, (B) Cavernicolins, (C) Hydroverongiaquinols, (D) Bromotyrosineketals, (E) Bromotyrosine Lactone derivatives, (F) Oxazolidones, (G) Spiroisoxazolines, (H) Verongiabenzenoids, (I) Verongiaquinols and (J) Dibromocyclohexadiens.

In view of their potential for producing new compounds of pharmacological interest, sponges have been one of the most studied organisms from a chemical point of view. Over the past 20 years, hundreds of substances have been isolated from sponges, many of which have been identified and show interesting biological and pharmacological activities, as for example, antibacterial, anticoagulant, antifungal, anti-inflammatory, antimalarial, antiplatelet, antituberculosis, antiviral, immunosuppressive and neurosuppressive activities.

The species of the genus Aplysina also show a wide structural variety of nitrogen compounds, present only in marine sponges. Therefore they are a rich source for research of new structural models for future therapeutic applications. With the information provided in this review, we hope to facilitate research in the field and to contribute to a better understanding and knowledge of the phytochemistry of this genus.

\section{Acknowledgements}

The authors thank the University of Illinois in Chicago, U.S.A., for the use of the NAPRALERT database for this study. Financial support was provided by $\mathrm{CNPq}$ (Conselho Nacional de Desenvolvimento Científico e Tecnológico), PgPNSB (Pós-Graduação em Produtos Naturais e Sintéticos Bioativos), and PRONEX-FAPESQ-PB (Programa de Apoio a Núcleos de Excelência-Fundação de Apoio a Pesquisa do Estado da Paraíba).We also thank Mr. Raimundo Nonato da Silva Filho for technical help.

\section{References}

1. Sponge. Available online: http://www.britannica.com (accessed on 5 October 2010).

2. Hentschel, U.; Hopke, J.; Horn, M.; Friedrich, A.B.; Wagner, M.; Hacker, J.; Moore, B.S. Molecular evidence for a uniform microbial community in sponges from different oceans. Appl. Environ. Microbiol. 2002, 68, 4431-4440.

3. Radjasa, O.K.; Sabdono, A.; Junaidi, J.; Zocchi, E. Richness of secondary metabolite-producing marine bacteria associated with sponge Haliclona sp. Int. J. Pharmacol. 2007, 3, 275-279.

4. Thomas, T.R.A.; Kavlekar, D.P.; LokaBharathi, P.A. Marine drugs from sponge-microbe association-a review. Mar. Drugs 2010, 8, 1417-1468.

5. Ebada, S.S.; Lin, W.; Proksch, P. Bioactive sesterterpenes and triterpenes from marine sponges: Occurrence and pharmacological significance. Mar. Drugs 2010, 8, 313-346. 
6. Andavan, G.S.B.; Lemmens-Gruber, R. Cyclodepsipeptides from marine sponges: Natural agents for drug research. Mar. Drugs 2010, 8, 810-834.

7. Balan, V.; Nangia-Makker, P.; Raz, A. Galectins as cancer biomarkers. Cancers 2010, 2, 592-610.

8. Exposito, J.-Y.; Valcourt, U.; Cluzel, C.; Lethias, C. The fibrillar collagen family. Int. J. Mol. Sci. 2010, 11, 407-426.

9. Longeon, A.; Copp, B.R.; Quévrain, E.; Roué, M.; Kientz, B.; Cresteil, T.; Petek, S.; Debitus, C.; Bourguet-Kondracki, M.-L. Bioactive indole derivatives from the south pacific marine sponges Rhopaloeides odorabile and Hyrtios sp. Mar. Drugs 2011, 9, 879-888.

10. Lee, Y.K.; Lee, J.-H.; Lee, H.K. Microbial symbiosis in marine sponges. J. Microbiol. 2001, 39, 254-264.

11. Belarbi, E.H.; Gómez, A.C.; Chisti, Y.; Camacho, F.G.; Grima, E.M. Producing drugs from marine sponges. Biotechnol. Adv. 2003, 21, 585-598.

12. Fieseler, L.; Horn, M.; Wagner, M.; Hentschel, U. Discovery of the novel candidate phylum "Poribacteria" in marine sponges. Appl. Environ. Microbiol. 2004, 70, 3724-3732.

13. Thoms, C.; Ebel, R.; Proksch, P. Activated chemical defense in Aplysina sponges revisited. J. Chem. Ecol. 2006, 32, 97-123.

14. Thakur, N.L.; Müller, W.E.G. Biotechnological potential of marine sponges. Curr. Sci. India 2004, 86, 1506-1512.

15. Erpenbeck, D.; van Soest, R. Status and perspective of sponge chemosystematics. Mar. Biotechnol. 2007, 9, 2-19.

16. Fusetani, N.; Matsunaga, S. Bioactive sponge peptides. Chem. Rev. 1993, 93, 1793-1806.

17. Sagar, S.; Kaur, M.; Minneman, K.P. Antiviral lead compounds from marine sponges. Mar. Drugs 2010, 8, 2619-2638.

18. Koopmans, M.; Martens, D.; Wijffels, R. Towards commercial production of sponge medicines. Mar. Drugs 2009, 7, 787-802.

19. Sepčić, K.; Kauferstein, S.; Mebs, D.; Turk, T. Biological activities of aqueous and organic extracts from tropical marine sponges. Mar. Drugs 2010, 8, 1550-1566.

20. Esteves, A.I.S.; Nicolai, M.; Humanes, M.; Goncalves, J. Sulfated polysaccharides in marine sponges: Extraction methods and anti-HIV activity. Mar. Drugs 2011, 9, 139-153.

21. Alvarez, B.; Crisp, M.D.; Driver, F.; Hooper, J.N.A.; van Soest, R.W.M. Phylogenetic relationships of the family Axinellidae (Porifera: Demospongiae) using morphological and molecular data. Zool. Scr. 2000, 29, 169-198.

22. Proksch, P.; Edrada, R.; Ebel, R. Drugs from the seas - Current status and microbiological implications. Appl. Microbiol. Biot. 2002, 59, 125-134.

23. Sipkema, D.; Franssen, M.; Osinga, R.; Tramper, J.; Wijffels, R. Marine sponges as pharmacy. Mar. Biotechnol. 2005, 7, 142-162.

24. Tymiak, A.A.; Rinehart, K.L., Jr.; Bakus, G.J. Constituents of morphologically similar sponges: Aplysina and Smenospongia species. Tetrahedron 1985, 41, 1039-1047.

25. Rinehart, K.L., Jr.; Shaw, P.D.; Shield, L.S.; Gloer, J.B.; Harbour, G.C.; Koker, M.E.S.; Samain, D.; Schwartz, R.E.; Tymiak, A.A.; Weller, D.L.; et al. Marine natural products as sources of antiviral, antimicrobial and antineoplastic agents. Pure Appl. Chem. 1981, 53, 795-817. 
26. Goo, Y.M. Antimicrobial and antineoplastic tyrosine metabolites from a marine sponge, Aplysina fistularis. Arch. Pharm. Res. 1985, 8, 21-30.

27. Capon, R.; Macleod, J. Two epimeric dibromo nitriles from the Australian sponge Aplysina laevis. Aust. J. Chem. 1987, 40, 341-346.

28. Ajabnoor, M.A.M.; Tilmisany, A.K.; Taha, A.M.; Antonius, A. Isolation of antibiotic principles from some red sea sponges. Egypt. J. Pharm. Sci. 1991, 32, 797-815.

29. Gunasekera, S.P.; Cross, S.S. Fistularin 3 and 11-ketofistularin 3. feline leukemia virus active bromotyrosine metabolites from the marine sponge Aplysina archeri. J. Nat. Prod. 1992, 55, 509-512.

30. Rodríguez, A.D.; Piña, I.C. The structures of aplysinamisines I, II, and III: New bromotyrosine-derived alkaloids from the Caribbean sponge Aplysina cauliformis. J. Nat. Prod. 1993, 56, 907-914.

31. Gopichand, Y.; Schmitz, F.J. Marine natural products: Fistularin-1, -2 and -3 from the sponge Aplysina fistularis forma fulva. Tetrahedron Lett. 1979, 20, 3921-3924.

32. Ajabnoor, M.A.M.; Tilmisany, A.K.; Taha, A.M.; Antonius, A. Effect of red sea sponge extracts on blood glucose levels in normal mice. J. Ethnopharmacol. 1991, 33, 103-106.

33. Pinheiro, U.S.; Hajdu, E.; Custódio, M.R. Aplysina nardo (Porifera, Verongida, Aplysinidae) from the Brazilian coast with description of eight new species. Zootaxa 2007, 1609, 1-51.

34. Ciminiello, P.; Costantino, V.; Fattorusso, E.; Magno, S.; Mangoni, A.; Pansini, M. Chemistry of Verongida sponges, II. Constituents of the Caribbean sponge Aplysina fistularis forma fulva. J. Nat. Prod. 1994, 57, 705-712.

35. Pauletti, P.M.; Cintra, L.S.; Braguine, C.G.; Filho, A.A.S.; Silva, M.L.A.; Cunha, W.R.; Januário, A.H. Halogenated indole alkaloids from marine invertebrates. Mar. Drugs 2010, 8 , $1526-1549$.

36. Aiello, A.; Fattorusso, E.; Imperatore, C.; Menna, M.; Müller, W. Iodocionin, a cytotoxic iodinated metabolite from the Mediterranean Ascidian Ciona edwardsii. Mar. Drugs 2010, 8, 285-291.

37. Cabrita, M.T.; Vale, C.; Rauter, A.P. Halogenated compounds from marine algae. Mar. Drugs 2010, 8, 2301-2317.

38. Vairappan, C.S.; Ishii, T.; Lee, T.K.; Suzuki, M.; Zhaoqi, Z. Antibacterial activities of a new brominated diterpene from Borneon Laurencia spp. Mar. Drugs 2010, 8, 1743-1749.

39. La Barre, S.; Potin, P.; Leblanc, C.; Delage, L. The Halogenated metabolism of brown algae (Phaeophyta), its biological importance and its environmental significance. Mar. Drugs 2010, 8, 988-1010.

40. Güven, K.C.; Percot, A.; Sezik, E. Alkaloids in marine algae. Mar. Drugs 2010, 8, 269-284.

41. Genovese, G.; Tedone, L.; Hamann, M.; Morabito, M. The Mediterranean red alga Asparagopsis: A source of compounds against Leishmania. Mar. Drugs 2009, 7, 361-366.

42. Matta, C.B.B.; Souza, E.T.; Queiroz, A.C.; Lira, D.P.; Araújo, M.V.; Cavalcante-Silva, L.H.A.; Miranda, G.E.C.; Araújo-Júnior, J.X.; Barbosa-Filho, J.M.; Santos, B.V.O.; et al. Antinociceptive and anti-inflammatory activity from algae of the genus Caulerpa. Mar. Drugs 2011, 9, 307-318. 
43. Almeida, C.L.F.; Falcão, H.S.; Lima, G.R.M.; Montenegro, C.A.; Lira, N.S.; Athayde-Filho, P.F.; Rodrigues, L.C.; Souza, M.F.V.; Barbosa-Filho, J.M.; Batista, L.M. Bioactivities from marine algae of the genus Gracilaria. Int. J. Mol. Sci. 2011, 12, 4550-4573.

44. Souza, E.T.; Lira, D.P.; Queiroz, A.C.; Silva, D.J.C.; Aquino, A.B.; Mella, E.A.C.; Lorenzo, V.P.; Miranda, G.E.C.; Araújo-Júnior, J.X.; Chaves, M.C.O.; et al. The Antinociceptive and anti-inflammatory activities of caulerpin, a bisindole alkaloid isolated from seaweeds of the genus Caulerpa. Mar. Drugs 2009, 7, 689-704.

45. Queiroz, T.M.; Machado, N.T.; Furtado, F.F.; Oliveira-Filho, A.A.; Alustau, M.C.; Figueiredo, C.S.; Miranda, G.E.C.; Barbosa-Filho, J.M.; Braga, V.A.; Medeiros, I.A.; Vasorelaxation induced by Dictyota pulchella (Dictyotaceae), a brown alga, is mediated via inhibition of calcium influx in rats. Mar. Drugs 2011, 9, 2075-2088.

46. Lira, N.S.; Monte-Neto, R.L.; Marchi, J.G.B.; Lins, A.C.S.; Pinheiro, U.S.; Tavares, J.F.; Silva, M.S.; Cunha, E.V.L.; Braz Filho, R.; Santos, C.F.; et al. Aplysfistularine: A novel dibromotyrosine derivative isolated from Aplysina fistularis. Mar. Drugs 2011, submitted.

47. Lúcio, A.S.S.C.; Almeida, J.R.G.S.; Barbosa-Filho, J.M.; Pita, J.C.L.R.; Castello Branco, M.V.S.; Diniz, M.F.F.M.; Agra, M.F.; Cunha, E.V.L.; Silva, M.S.; Tavares, J.F. Azaphenathrene alkaloids with antitumoral activity from Anaxagorea dolichocarpa Sprague \& Sandwith (Annonaceae). Molecules 2011, 16, 7125-7131.

48. Bitencourt, M.A.O.; Dantas, G.R.; Lira, D.P.; Barbosa-Filho, J.M.; Miranda, G.E.C.; Santos, B.V.O.; Souto, J.T. Aqueous and methanolic extracts of Caulerpa mexicana suppress cell migration and ear edema induced by inflammatory agents. Mar. Drugs 2011, 9, 1332-1345.

49. Alves, J.S.; Castro, J.C.M.; Freire, M.O.; da-Cunha, E.V.L.; Barbosa-Filho, J.M.; Silva, M.S. Complete assignment of the ${ }^{1} \mathrm{H}$ and ${ }^{13} \mathrm{C}$ NMR spectra of four triterpenes of the ursane, artane, lupane and friedelane groups. Magn. Reson. Chem. 2000, 38, 201-206.

50. Andrade, N.C.; Cunha, E.V.L.; Silva, M.S.; Agra, M.F.; Barbosa-Filho, J.M. Terpenoids of the Annonaceae: Distribution and Compilation of ${ }^{13} \mathrm{C}$ NMR Data. In Recent Research Developments in Phytochemistry; Pandalai, S.G., Ed.; Research Signpost: Kerala, India, 2003; Volume 7, pp. 1-85.

51. Sena-Filho, J.G.; Duringer, J.; Maia, G.L.A.; Tavares, J.F.; Xavier, H.S.; Silva, M.S.; da-Cunha, E.V.L.; Barbosa-Filho, J.M. Ecdysteroids from Vitex species: Distribution and compilation of their ${ }^{13} \mathrm{C}-\mathrm{NMR}$ spectral data. Chem. Biodivers. 2008, 5, 707-713.

52. Oliveira, S.L.; Silva, M.S.; Tavares, J.F.; Sena-Filho, J.G.; Lucena, H.F.S.; Romero, M.A.V.; Barbosa-Filho, J.M. Tropane alkaloids from Erythroxylum genus: Distribution and compilation of ${ }^{13} \mathrm{C}$-NMR spectral data. Chem. Biodivers. 2010, 7, 302-326.

53. Almeida, R.N.; Navarro, D.S.; Barbosa-Filho, J.M. Plants with central analgesic activity. Phytomedicine 2001, 8, 310-322.

54. Silva, J.S.; Moura, M.D.; Oliveira, R.A.G.; Diniz, M.F.F.; Barbosa-Filho, J.M. Natural product inhibitors of ovarian neoplasia. Phytomedicine 2003, 10, 221-232.

55. Falcão, H.S.; Mariath, I.R.; Diniz, M.F.F.M.; Batista, L.M.; Barbosa-Filho, J.M. Plants of the American continent with antiulcer activity. Phytomedicine 2008, 15, 132-146. 
56. Sousa, F.C.F.; Melo, C.T.V.; Citó, M.C.O.; Félix, F.H.C.; Vasconcelos, S.M.M.; Fonteles, M.M.F.; Barbosa Filho, J.M.; Viana, G.S.B. Plantas medicinais e seus constituintes bioativos: Uma revisão da bioatividade e potenciais benefícios nos distúrbios da ansiedade em modelos animais. Rev. Bras. Farmacogn. 2008, 18, 642-654.

57. Falcão, H.S.; Leite, J.A.; Barbosa-Filho, J.M.; Athayde-Filho, P.F.; Chaves, M.C.O.; Moura, M.D.; Ferreira, A.L.; Almeida, A.B.A.; Souza-Brito, A.R.M.; Diniz, M.F.F.M.; et al. Gastric and duodenal antiulcer activity of alkaloids: A review. Molecules 2008, 13, 3198-3223.

58. Mota, K.S.L.; Dias, G.E.N.; Pinto, M.E.F.; Luiz-Ferreira, A.; Souza-Brito, A.R.S.; Hiruma-Lima, C.A.; Barbosa-Filho, J.M.; Batista, L.M. Flavonoids with gastroprotective activity. Molecules 2009, 14, 979-1012.

59. Mariath, I.R.; Falcão, H.S.; Barbosa-Filho, J.M.; Sousa, L.C.F.; Tomaz, A.C.A.; Batista, L.M.; Diniz, M.F.F.M.; Athayde-Filho, P.F.; Tavares, J.F.; Silva, M.S.; et al. Plants of the American continent with antimalarial activity. Rev. Bras. Farmacogn. 2009, 19, 158-191.

60. Ribeiro Filho, J.; Falcao, H.S.; Batista, L.M.; Barbosa-Filho, J.M.; Piuvezam, M.R. Effects of Plant Extracts on HIV-1 Protease. Curr. HIV Res. 2010, 8, 531-544.

61. Lima, G.R.M.; Montenegro, C.A.; Almeida, C.L.F.; Athayde-Filho, P.F.; Barbosa-Filho, J.M.; Batista, L.M. Database survey of anti-inflammatory plants in South America: A review. Int. J. Mol. Sci. 2011, 12, 2692-2749.

62. Silva, F.L.; Fischer, D.C.H.; Tavares, J.F.; Silva, M.S.; Athayde-Filho, P.F.; Barbosa-Filho, J.M. Compilation of secondary metabolites from Bidens pilosa L. Molecules 2011, 16, 1070-1102.

63. Ciminiello, P.; Fattorusso, E.; Forino, M.; Magno, S.; Pansini, M. Chemistry of verongida sponges VIII-bromocompounds from the Mediterranean sponges Aplysina aerophoba and Aplysina cavernicola. Tetrahedron 1997, 53, 6565-6572.

64. Norte, M.; Rodriguez, M.L.; Fernández, J.J.; Eguren, L.; Estrada, D.M. Aplysinadiene and $(R, R) 5$ [3,5-dibromo-4-[(2-oxo-5-oxazolidinyl)] methoxyphenyl]-2-oxazolidinone, two novel metabolites from Aplysina aerophoba Synthes. Tetrahedron 1988, 44, 4973-4980.

65. Borders, D.B.; Morton, G.O.; Wetzel, E.R. Structure of a novel bromine compound isolated from a sponge. Tetrahedron Lett. 1974, 15, 2709-2712.

66. Fattorusso, E.; Minale, L.; Sodano, G. Aeroplysinin-1, a new bromo-compound from Aplysina aerophoba. J. Chem. Soc. Chem. Comm. 1970, 751-752.

67. Cimino, G.; De Rosa, S.; De Stefano, S.; Self, R.; Sodano, G. The bromo-compounds of the true sponge Verongia aerophoba. Tetrahedron Lett. 1983, 24, 3029-3032.

68. Gunasekera, M.; Gunasekera, S.P. Dihydroxyaerothionin and aerophobin 1. Two brominated tyrosine metabolites from the deep water marine sponge Verongula rigida. J. Nat. Prod. 1989, $52,753-756$.

69. Kelecom, A.; Kannengiesser, G.J. Chemical constituents of Verongia sponges, II-Struture of two dibrominated compounds isolated from the Mediterranean sponge Verongia aerophoba. Ann. Acad. Braz. Sci. 1979, 51, 639-641.

70. Fattorusso, E.; Minale, L.; Sodano, G. Aeroplysinin-1, an antibacterial bromo-compound from the sponge Verongia aerophoba. J. Chem. Soc. Perk. Trans. 1 1972, 16-18. 
71. Makarieva, T.N.; Stonik, V.A.; Alcolado, P.; Elyakov, Y.B. Comparative study of the halogenated tyrosine derivatives from Demospongiae (Porifera). Comp. Biochem. Phys. B 1981, $68,481-484$.

72. Mancini, I.; Guella, G.; Laboute, P.; Debitus, C.; Pietra, F. Hemifistularin 3: A degraded peptide or biogenetic precursor? Isolation from a sponge of the order Verongida from the coral sea or generation from base treatment of 11-oxofistularin 3. J. Chem. Soc. Perk. Trans. 1 1993, 3121-3125.

73. Ciminiello, P.; Dell'Aversano, C.; Fattorusso, E.; Magno, S. Archerine, a novel anti-histaminic bromotyrosine-derived compound from the Caribbean marine sponge Aplysina archeri. Eur. J. Org. Chem. 2001, 2001, 55-60.

74. Ciminiello, P.; Dell'Aversano, C.; Fattorusso, E.; Magno, S.; Carrano, L.; Pansini, M. Chemistry of Verongida sponges. VII bromocompounds from the Caribbean sponge Aplysina archeri. Tetrahedron 1996, 52, 9863-9868.

75. Saeki, B.M.; Granato, A.C.; Berlinck, R.G.S.; Magalhães, A.; Schefer, A.B.; Ferreira, A.G.; Pinheiro, U.S.; Hajdu, E. Two unprecedented dibromotyrosine-derived alkaloids from the Brazilian endemic marine sponge Aplysina caissara. J. Nat. Prod. 2002, 65, 796-799.

76. Lira, T.O.; Berlinck, R.G.S.; Nascimento, G.G.F.; Hajdu, E. Further dibromotyrosine-derived metabolites from the marine sponge Aplysina caissara. J. Braz. Chem. Soc. 2006, 17, 1233-1240.

77. Rodríguez, A.D.; Akee, R.K.; Scheuer, P.J. Two bromotyrosine-cysteine derived metabolites from a sponge. Tetrahedron Lett. 1987, 28, 4989-4992.

78. Ciminiello, P.; Dell'Aversano, C.; Fattorusso, E.; Magno, S.; Pansini, M. Chemistry of Verongida sponges. 9. Secondary metabolite composition of the Caribbean sponge Aplysina cauliformis. J. Nat. Prod. 1999, 62, 590-593.

79. D'Ambrosio, M.; Guerriero, A.; Traldi, P.; Pietra, F. Cavernicolin-1 and cavernicolin-2, epimeric dibromolactams from the Mediterranean sponge Aplysina (Verongia) cavernicola. Tetrahedron Lett. 1982, 23, 4403-4406.

80. D'Ambrosio, M.; Guerriero, A.; de Clauser, R.; de Stanchina, G.; Pietra, F. Dichloroverongiaquinol, a new marine antibacterial compound from Aplysina cavernicola. Isolation and synthesis. Cell. Mol. Life Sci. 1983, 39, 1091-1092.

81. D’Ambrosio, M.; Guerriero, A.; Pietra, F. Novel, racemic or nearly-racemic antibacterial bromoand chloroquinols and $\gamma$-lactams of the verongiaquinol and the cavernicolin type from the marine sponge Aplysina (=Verongia) cavernicola. Helv. Chim. Acta 1984, 67, 1484-1492.

82. D’Ambrosio, M.; Mealli, C.; Guerriero, A.; Pietra, F. 7-Bromocavernicolenone, a new $\alpha$-bromoenone from the Mediterranean sponge Aplysina (=Verongia) cavernicola. Implied, unprecedented involvement of a halogenated dopa in the biogenesis of a natural product. Helv. Chim. Acta 1985, 68, 1453-1460.

83. Pereira, F.R.; Marques, S.O.; Nascimento, A.M.; Gandolfi, R.C.; Hajdu, E.; Peixinho, S.; Custódio, M.R.; Berlinck, R.G.S. Metabólitos Derivados da Dibromotirosina das Esponjas Aplysina fulva, A. conulosa (sp. n.) and A. fistularis. In XXIX Reunião Anual da Sociedade Brasileira de Química; Sociedade Brasileira de Química: Águas de Lindóia, Brazil, 2006; p. 335.

84. Xynas, R.; Capon, R.J. Two new bromotyrosine-derived metabolites from an Australian marine sponge, Aplysina sp. Aust. J. Chem. 1989, 42, 1427-1433. 
85. Tymiak, A.A. Structure elucidation and biosynthesis of selected bioactive marine natural products. Dissert. Abs. Int. B 1985, 43, 3990-3991.

86. Rinehart, K.L., Jr.; Johnson, R.D.; Paul, I.C.; McMillan, J.A.; Siuda, J.F.; Krejcarek, G.E. Identification of Compounds in Selected Marine Organisms by Gas Chromatography-Mass Spectrometry, Field Desorption Mass Spectrometry, and Other Physical Methods. In Food-Drugs from the Sea: Proceedings, 1974; Webber, H.H., Ruggieri, G.D., Eds.; Marine Technology Society: Columbia, MD, USA, 1976.

87. Sharma, G.M.; Vig, B.; Burkholder, P.R. Antimicrobial substances of sponges. IV. Structure of a bromine-containing compound from a marine sponge. J. Org. Chem. 1970, 35, 2823-2826.

88. Gandolfi, R.C.; Medina, M.B.; Berlinck, R.G.S.; Lira, S.P.; Galetti, F.C.S.; Silva, C.L.; Veloso, K.; Ferreira, A.G.; Hajdu, E.; Peixinho, S. Metabólitos secundários das esponjas Aplysina fistularis e Dysidea sp. e atividade antituberculose da 11-cetofistularina-3. Quim. Nova 2010, 33, 1853-1858.

89. Walker, R.P.; Thompson, J.E.; Faulkner, D.J. Exudation of biologically-active metabolites in the sponge Aplysina fistularis. II. Chemical evidence. Mar. Biol. 1985, 88, 27-32.

90. Kobayashi, J.I.; Tsuda, M.; Agemi, K.; Shigemori, H.; Ishibashi, M.; Sasaki, T.; Mikami, Y. Purealidins $\mathrm{B}$ and $\mathrm{C}$, new bromotyrosine alkaloids from the Okinawan marine sponge Psammaplysilla purea. Tetrahedron 1991, 47, 6617-6622.

91. Krejcarek, G.E.; White, R.H.; Hager, L.P.; McClure, W.O.; Johnson, R.D.; Rinehart, K.L.; McMillan, J.A.; Paul, I.C.; Shaw, P.D.; Brusca, R.C. A rearranged dibromotyrosine metabolite from Verongia aurea. Tetrahedron Lett. 1975, 16, 507-510.

92. Sharma, G.M.; Burkholder, P.R. Studies on antimicrobial substances of sponges. I. Isolation, purification, and properties of a new bromine-containing antibacterial substance. J. Antibiot. 1967, 20, 200-203.

93. Sharma, G.M.; Burkholder, P.R. Studies on the antimicrobial substances of sponges II. Structure and synthesis of a bromine-containing antibacterial, compound from a marine sponge. Tetrahedron Lett. 1967, 8, 4147-4150.

94. Minale, L. Natural product chemistry of the marine sponges. Pure Appl. Chem. 1976, 48, 7-23.

95. Minale, L.; Cimino, G.; de Stefano, S.; Sodano, G. Natural products from Porifera. Fortschr. Chem. Org. Naturst. 1976, 33, 1-72.

96. Carney, J.R.; Rinehart, K.L. Biosynthesis of brominated tyrosine metabolites by Aplysina fistularis. J. Nat. Prod. 1995, 58, 971-985.

97. Nuñez, C.V.; Almeida, E.V.R.; Granato, A.C.; Marques, S.O.; Santos, K.O.; Pereira, F.R.; Macedo, M.L.; Ferreira, A.G.; Hajdu, E.; Pinheiro, U.S.; et al. Chemical variability within the marine sponge Aplysina fulva. Biochem. Syst. Ecol. 2008, 36, 283-296.

98. Rogers, E.W.; Oliveira, M.F.; Berlinck, R.G.S.; König, G.M.; Molinski, T.F. Stereochemical heterogeneity in Verongid sponge metabolites. absolute stereochemistry of $(+)$-fistularin-3 and (+)-11-epi-fistularin-3 by microscale LCMS-Marfey's analysis. J. Nat. Prod. 2005, 68, 891-896.

99. Rogers, E.W.; Molinski, T.F. Highly polar spiroisoxazolines from the sponge Aplysina fulva. J. Nat. Prod. 2007, 70, 1191-1194.

100. Encarnación, R.D.; Sandoval, E.; Malmstrøm, J.; Christophersen, C. Calafianin, a bromotyrosine derivative from the marine sponge Aplysina gerardogreeni. J. Nat. Prod. 2000, 63, 874-875. 
101. Hernández-Guerrero, C.J.; Zubía, E.; Ortega, M.J.; Carballo, J.L. Cytotoxic dibromotyrosine-derived metabolites from the sponge Aplysina gerardogreeni. Bioorg. Med. Chem. 2007, 15, 5275-5282.

102. Fendert, T.; Wray, V.; van Soest, R.W.M.; Proksch, P. Bromoisoxazoline alkaloids from the Caribbean sponge Aplysina insularis. Z. Naturforsch. 1999, 54c, 246-252.

103. Compagnone, R.S.; Avila, R.; Suárez, A.I.; Abrams, O.V.; Rangel, H.R.; Arvelo, F.; Piña, I.C.; Merentes, E. 11-Deoxyfistularin-3, a new cytotoxic metabolite from the Caribbean sponge Aplysina fistularis insularis. J. Nat. Prod. 1999, 62, 1443-1444.

104. Acosta, A.L.; Rodríguez, A.D. 11-Oxoaerothionin: A cytotoxic antitumor bromotyrosine-derived alkaloid from the Caribbean marine sponge Aplysina lacunosa. J. Nat. Prod. 1992, 55, 1007-1012.

105. Evan, T.; Rudi, A.; Ilan, M.; Kashman, Y. Aplyzanzine A, a new dibromotyrosine derivative from a Verongida sponge. J. Nat. Prod. 2001, 64, 226-227.

106. Kossuga, M.H.; Lira, S.P.; Nascimento, A.M.; Gambardella, M.T.P.; Berlinck, R.G.S.; Torres, Y.R.; Nascimento, G.G.F.; Pimenta, E.F.; Silva, M.; Thiemann, O.H.; et al. Isolamento e atividades biológicas de produtos naturais das esponjas Monanchora arbuscula, Aplysina sp. Petromica ciocalyptoides e Topsentia ophiraphidites, da ascídia Didemnum ligulum e do octocoral Carijoa riisei. Quim. Nova 2007, 30, 1194-1202.

107. Kashman, Y.; Groweiss, A.; Carmely, S.; Kinamoni, Z.; Czarkie, D.; Rotem, M. Recent research in marine natural products from the red sea. Pure Appl. Chem. 1982, 54, 1995-2010.

108. Ciminiello, P.; Dell'Aversano, C.; Fattorusso, E.; Magno, S.; Pansini, M. Chemistry of Verongida sponges. 10. Secondary metabolite composition of the Caribbean Sponge Verongula gigantea. J. Nat. Prod. 2000, 63, 263-266.

109. Cruz, F.; Quijano, L.; Gómez-Garibay, F.; Rios, T. Brominated metabolites from the sponge Aplysina (Verongia) thiona. J. Nat. Prod. 1990, 53, 543-548.

110. Ayanoglu, E.; Djerassi, C.; Erdman, T.R.; Scheuer, P.J. Minor and trace sterols in marine invertebrates V. isolation, structure elucidation and synthesis of 3[beta]-hydroxy-26,27bisnorcholest-5-en-24-one from the sponge Psammaplysilla purpurea. Steroids 1978, 31, $815-822$.

111. Carballeira, N.M.; Bou, C.R. The sterol composition of the marine sponge Aplysina (=Verongia) archeri: A comparative study of the Verongidae. Comp. Biochem. Phys. B 1989, 93, 175-179.

112. Hertzberg, S.; Bergquist, P.; Liaaen-Jensen, S. Further occurrence of sulphated carotenoids in Ianthella species (Desmospongia). Biochem. Syst. Ecol. 1989, 17, 51-53.

113. Nichols, S.A. An evaluation of support for order-level monophyly and interrelationships within the class Demospongiae using partial data from the large subunit rDNA and cytochrome oxidase subunit I. Mol. Phylogenet. Evol. 2005, 34, 81-96.

114. Lawson, M.; Thompson, J.; Djerassi, C. Localization of long-chain fatty acids and unconventional sterols in spherulous cells of a marine sponge. Lipids 1988, 23, 1037-1048.

115. Ciminiello, P.; Fattorusso, E.; Magno, S.; Pansini, M. Chemistry of Verongida sponges VI. Comparison of the secondary metabolic composition of Aplysina insularis and Aplysina fulva. Biochem. Syst. Ecol. 1996, 24, 105-113. 
116. Hamann, M.T.; Scheuer, P.J.; Kelly-Borges, M. Biogenetically diverse, bioactive constituents of a sponge, order Verongida: Bromotyramines and sesquiterpene-shikimate derived metabolites. J. Org. Chem. 1993, 58, 6565-6569.

117. D'Ambrosio, M.; Guerriero, A.; Pietra, F. N-Carbamoylpyrrolidine and 7-chlorocavernicolenone, two new metabolites of the Mediterranean sponge Aplysina (=Verongia) cavernicola. Comp. Biochem. Phys. B 1986, 83, 309-312.

118. Cavazza, M.; Guella, G.; Nucci, L.; Pergola, F.; Bicchierini, N.; Pietra, F. Anodic oxidation of 3,5-dihalogenotyrosines as a model reaction for the biogenesis of the cavernicolins, metabolites of the Verongid sponge Aplysina cavernicola. J. Chem. Soc. Perk. Trans. 1 1993, 3117-3120.

119. Calcul, L.; Inman, W.D.; Morris, A.A.; Tenney, K.; Ratnam, J.; McKerrow, J.H.; Valeriote, F.A.; Crews, P. Additional insights on the bastadins: Isolation of analogues from the sponge Ianthella cf. reticulata and exploration of the oxime configurations. J. Nat. Prod. 2010, 73, 365-372.

120. Andersen, R.J.; Faulkner, D.J. A novel antibiotic from a sponge of the genus Verongia. Tetrahedron Lett. 1973, 14, 1175-1178.

121. Kasperek, G.J.; Bruice, T.C.; Yagi, H.; Kaubsich, N.; Jerina, D.M. Solvolytic chemistry of 1,4-dimethylbenzene oxide. A new and novel mechanism for the NIH shift. J. Am. Chem. Soc. 1972, 94, 7876-7882.

122. Huang, X.-P.; Deng, Z.-W.; van Soest, R.W.M.; Lin, W.-H. Brominated derivatives from the Chinese sponge Pseudoceratina sp. J. Asian Nat. Prod. Res. 2008, 10, 239-242.

123. Kossuga, M.H.; MacMillan, J.B.; Rogers, E.W.; Molinski, T.F.; Nascimento, G.G.F.; Rocha, R.M.; Berlinck, R.G.S. (2S,3R)-2-Aminododecan-3-ol, a new antifungal agent from the Ascidian Clavelina oblonga. J. Nat. Prod. 2004, 67, 1879-1881.

124. Peng, J.; Li, J.; Hamann, M.T. The Marine Bromotyrosine Derivatives. In The Alkaloids: Chemistry and Biology; Cordell, G.A., Ed.; Academic Press: Gulf Breeze, FL, USA, 2005; Volume 61, p. 59.

125. Aiello, A.; Fattorusso, E.; Menna, M.; Pansini, M. Chemistry of Verongida sponges-V. Brominated metabolites from the Caribbean sponge Pseudoceratina sp. Biochem. Syst. Ecol. 1995, 23, 377-381.

126. Moody, K.; Thomson, R.H.; Fattorusso, E.; Minale, L.; Sodano, G. Aerothionin and homoaerothionin: Two tetrabromo spirocyclohexadienylisoxazoles from Verongia sponges. J. Chem. Soc. Perk. Trans 1 1972, 1, 18-24.

127. Bergquist, P.R. Sponges; University of California Press: Berkeley and Los Angeles, CA, USA, 1978.

128. Teeyapant, R.; Proksch, P. Biotransformation of brominated compounds in the marine sponge Verongia aerophoba-Evidence for an induced chemical defense? Naturwissenschaften 1993, 80, 369-370.

129. Ebel, R.; Brenzinger, M.; Kunze, A.; Gross, H.J.; Proksch, P. Wound Activation of protoxins in marine sponge Aplysina aerophoba. J. Chem. Ecol. 1997, 23, 1451-1462.

130. Puyana, M.; Fenical, W.; Pawlik, J.R. Are there activated chemical defenses in sponges of the genus Aplysina from the Caribbean? Mar. Ecol. Prog. Ser. 2003, 246, 127-135.

131. Weiss, B.; Ebel, R.; Elbrächter, M.; Kirchner, M.; Proksch, P. Defense metabolites from the marine sponge Verongia aerophoba. Biochem. Syst. Ecol. 1996, 24, 1-7. 
132. Koulman, A.; Proksch, P.; Ebel, R.; Beekman, A.C.; van Uden, W.; Konings, A.W.T.; Pedersen, J.A.; Pras, N.; Woerdenbag, H.J. Cytoxicity and mode of action of aeroplysinin-1 and a related dienone from the sponge Aplysina aerophoba. J. Nat. Prod. 1996, 59, 591-594.

133. Nagaraja, K.V.; Shaw, P.D. Inhibition of wheat germ RNA polymerase II by 2,6-dibromobenzoquinone and related compounds from Aplysina fistularis. Arch. Biochem. Biophys. 1982, 215, 544-550.

134. Chang, C.W.J.; Weinheimer, A.J. 2-Hydroxy, 3,5-dibromo, 4-methoxyphenylacetamide. a dibromotyrosine metabolite from Psammopilla purpurea. Tetrahedron Lett. 1977, 18, 4005-4007.

135. Fulmor, W.; Van Lear, G.E.; Morton, G.O.; Mills, R.D. Isolation and absolute configuration of the aeroplysinin I enantiomorphic pair from Ianthella ardis. Tetrahedron Lett. 1970, 11, 4551-4552.

136. Gutiérrez, M.; Capson, T.L.; Guzmán, H.M.; González, J.; Ortega-Barría, E.; Quiñoá, E.; Riguera, R. Antiprotozoal activity against Plasmodium falciparum and Trypanosoma cruzi of aeroplysinin-1 isolated from the new sponge Aplysina chiriquensis. Pharm. Biol. 2005, 43, $762-765$.

137. Córdoba, R.; Tormo, N.S.; Medarde, A.F.; Plumet, J. Antiangiogenic versus cytotoxic activity in analogues of aeroplysinin-1. Bioorg. Med. Chem. 2007, 15, 5300-5315.

138. Simões, C.M.O.; Petrovick, P.R. Farmacognosia: Da Planta ao Medicamento, 6 ed.; Editora da UFSC: Santa Catarina, Brasil, 2010.

(C) 2011 by the authors; licensee MDPI, Basel, Switzerland. This article is an open access article distributed under the terms and conditions of the Creative Commons Attribution license (http://creativecommons.org/licenses/by/3.0/). 